\title{
Large-Angle Anomalies in the CMB
}

\author{
Craig J. Copi, ${ }^{1}$ Dragan Huterer, ${ }^{2}$ Dominik J. Schwarz, ${ }^{3}$ and Glenn D. Starkman ${ }^{4}$ \\ ${ }^{1}$ CERCA, Department of Physics, Case Western Reserve University, Cleveland, OH 44106-7079, USA \\ ${ }^{2}$ Department of Physics, University of Michigan, 450 Church Street, Ann Arbor, MI 48109-1040, USA \\ ${ }^{3}$ Fakultät für Physik, Universität Bielefeld, Postfach 100131, 33501 Bielefeld, Germany \\ ${ }^{4}$ CERCA/ISO, Department of Physics, Case Western Reserve University, Cleveland, OH 44106-7079, USA
}

Correspondence should be addressed to Dragan Huterer, huterer@umich.edu

Received 30 April 2010; Revised 7 August 2010; Accepted 19 August 2010

Academic Editor: Eiichiro Komatsu

Copyright () 2010 Craig J. Copi et al. This is an open access article distributed under the Creative Commons Attribution License, which permits unrestricted use, distribution, and reproduction in any medium, provided the original work is properly cited.

We review the recently found large-scale anomalies in the maps of temperature anisotropies in the cosmic microwave background. These include alignments of the largest modes of $\mathrm{CMB}$ anisotropy with each other and with geometry and direction of motion of the solar ssystem, and the unusually low power at these largest scales. We discuss these findings in relation to expectation from standard inflationary cosmology, their statistical significance, the tools to study them, and the various attempts to explain them.

\section{Introduction: Why Large Scales are Interesting?}

The Copernican principle states that the Earth does not occupy a special place in the universe and that observations made from Earth can be taken to be broadly characteristic of what would be seen from any other point in the universe at the same epoch. The microwave sky is isotropic, apart from a Doppler dipole and a microwave foreground from the Milky Way. Together with the Copernican principle and some technical assumptions, an oft-inferred consequence is the socalled cosmological principle. It states that the distributions of matter and light in the Universe are homogeneous and isotropic at any epoch and thus also defines what we mean by cosmic time.

This set of assumptions is a crucial, implicit ingredient in obtaining most important results in quantitative cosmology, for example, it allows us to treat cosmic microwave background (CMB) temperature fluctuations in different directions in the sky as multiple probes of a single statistical ensemble, leading to the precision determinations of cosmological parameters that we have today.

Although we have some observational evidence that homogeneity and isotropy are reasonably good approximations to reality, neither of these are actual logical consequences of the Copernican principle, for example, the geometry of space could be homogeneous but anisotropiclike the surface of a sharp mountain ridge, with a gentle path ahead but the ground dropping steeply away to the sides. Indeed, three-dimensional space admits not just the three well known homogeneous isotropic geometries (Euclidean, spherical and hyperbolic- $E^{3}, S^{3}$, and $H^{3}$ ) but five others which are homogeneous but anisotropic. The two simplest are $S^{2} \times E^{1}$ and $H^{2} \times E^{1}$. These spaces support the cosmological principle but have preferred directions.

Similarly, although the Earth might not occupy a privileged place in the universe, it is not necessarily true that all points of observation are equivalent, for example, the topology of space may not be simply connected-we could live in a three dimensional generalization of a torus so that if you travel far enough in certain directions you come back to where you started. While such three-spaces generically admit locally homogeneous and isotropic geometries, certain directions or points might be singled out when nonlocal measurements are considered, for example the length of the shortest closed nontrivial geodesic through a point depends on the location of that point within the fundamental domain. Similarly, the inhomogeneity and anisotropy of eigenmodes of differential operators on such spaces are likely to translate into statistically inhomogeneous and anisotropic large scale structure, in the manner of Chladni figures on vibrating plates. 
The existence of nontrivial cosmic topology and of anisotropic geometry are questions that can only be answered observationally. In this regard, it is worth noting that our record at predicting the gross properties of the universe on large scales from first principles has been rather poor. According to the standard concordance model of cosmology, over $95 \%$ of the energy content of the universe is extraordinary-dark matter or dark energy whose existence has been inferred from the failure of the Standard Model of particle physics plus General Relativity to describe the behavior of astrophysical systems larger than a stellar cluster-while the very homogeneity and isotropy (and inhomogeneity) of the universe owe to the influence of an inflaton field whose particle-physics-identity is completely mysterious even after three decades of theorizing.

The stakes are set even higher with the recent discovery of dark energy that makes the universe undergo accelerated expansion. It is known that dark energy can affect the largest scales of the universe, for example, the clustering scale of dark energy may be about the horizon size today. Similarly, inflationary models can induce observable effects on the largest scales via either explicit or spontaneous violations of statistical isotropy. It is reasonable to suggest that statistical isotropy and homogeneity should be substantiated observationally, not just assumed. More generally, testing the cosmological principle should be one of the key goals of modern observational cosmology.

With the advent of high signal-to-noise maps of the cosmic microwave background anisotropies and with the conduct of nearly-full-sky deep galaxy surveys, statistical isotropy has begun to be precisely tested. Extraordinary full-sky temperature maps produced by the Wilkinson Microwave Anisotropy Probe (WMAP), in particular, are revolutionizing our ability to probe the universe on its largest scales [1-6]. In the near future, these will be joined by higher resolution temperature maps and high-resolution polarization maps and, eventually, by deep all-sky surveys, and perhaps by tomographic $21-\mathrm{cm}$ line observations that will extend our detailed knowledge of the universe's background geometry and fluctuations into the interior of the sphere of last scattering.

In this brief paper, we describe the large-scale anomalies in the CMB data, some of which were first reported on by the Cosmic Background Explorer (COBE) Differential Microwave Radiometer (DMR) collaboration in the mid 1990s. In particular, we report on alignments of the largest modes of $\mathrm{CMB}$ anisotropy with each other, and with geometry and direction of motion of the Solar System, as well as on unusually low angular correlations at the largest angular scales. We discuss these findings and, as this is not meant to be a comprehensive review and we emphasize results based on our own work in the area, we refer the reader to literature for all developments in the field. This paper extends an earlier review on the subject by Huterer [7], and complements another review on statistical isotropy in this special issue [8].

The paper is organized as follows. In Section 2 we describe the statistical quantities that describe the CMB and the expectations for their values in the currently favored

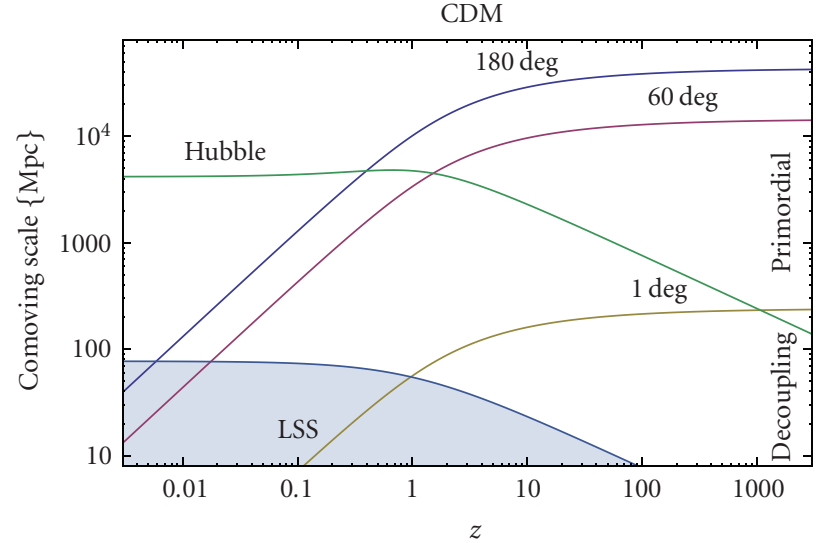

FIGURE 1: The comoving length within the context of the concordance model of an arc seen at a fixed angle and the comoving Hubble length as functions of redshift. Linear perturbation theory is expected to work well outside the shaded region, in which the large scale structure (LSS) forms.

$\Lambda \mathrm{CDM}$ cosmological model. In Section 3, we describe the alignments at the largest scales, as well as multipole vectors, which is a tool to study them. In Section 4, we describe findings of low power at largest scales in the CMB. Section 5 categorizes and covers the variety of possible explanations for these anomalies. We conclude in Section 6.

\section{Expectations from Cosmological Inflation}

A fixed angular scale on the sky probes the physics of the universe at a range of physical distances corresponding to the range of observable redshifts. This is illustrated in Figure 1, where the comoving lengths of arcs at fixed angle are shown as a function of redshift, together with the comoving Hubble scale. Angles of 1 degree and less probe events that were in causal contact at all epochs between the redshift of decoupling and today; this redshift range includes physical processes such as the secondary $\mathrm{CMB}$ anisotropies. The situation is different for angles $>60$ degrees, which subtend arcs that enter our Hubble patch only at $z \lesssim 1$. Therefore, the primordial CMB signal on such large angular scales could only be modified by the physics of local foregrounds and cosmology in the relatively recent past $(z \lesssim 1)$. Because they correspond to such large physical scales, the largest observable angular scales provide the most direct probe of the primordial fluctuations - whether generated during the epoch of cosmological inflation or preceding it.

2.1. Statistical Isotropy. What do we expect for the large angular scales of the CMB? A crucial ingredient of cosmology's concordance model is cosmological inflation-a period of accelerating cosmic expansion in the early universe. If we assume that inflationary expansion persisted for sufficiently many e-folds, then we expect to live in a homogeneous and isotropic universe within a domain larger than our Hubble volume. This homogeneity and isotropy will not be exact but should characterize both the background and 
the statistical distributions of matter and metric fluctuations around that background. These fluctuations are made visible as anisotropies of the CMB temperature and polarization, which are expected to inherit the underlying statistical isotropy. The temperature $T$ seen in direction $\hat{e}$ is predicted to be described by a Gaussian random field on the sky (i.e., the 2-sphere $S^{2}$ ), which implies that we can expand it in terms of spherical harmonics $Y_{\ell m}(\hat{e})$ multiplied by independent Gaussian random coefficients $a_{\ell m}$ of zero mean.

Statistical isotropy implies that the expectation values of all $n$-point correlation functions (of the temperature or polarization) are invariant under arbitrary rotations of the sky. As a consequence the expectation of the temperature coefficients is zero, $\left\langle a_{\ell m}\right\rangle=0$, for all $\ell>0$ and $m=-\ell,-\ell+$ $1, \ldots,+\ell$. The two-point correlation becomes a function of $\cos \theta \equiv \hat{e}_{1} \cdot \hat{e}_{2}$ only and can be expanded in terms of Legendre polynomials:

$$
\left\langle T\left(\hat{e}_{1}\right) T\left(\hat{e}_{2}\right)\right\rangle \equiv C(\theta)=\frac{1}{4 \pi} \sum_{\ell}(2 \ell+1) C_{\ell} P_{\ell}(\cos \theta) .
$$

Statistical independence implies that expectations of $a_{\ell m}$ with different $\ell$ and $m$ vanish. In particular, the two-point correlation function is diagonal in $\ell$ and $m$ :

$$
\left\langle a_{\ell m}^{*} a_{\ell^{\prime} m^{\prime}}\right\rangle \propto \delta_{\ell \ell^{\prime}} \delta_{m m^{\prime}} .
$$

Statistical isotropy adds that the constant of proportionality depends only on $\ell$, not $m$ :

$$
\left\langle a_{\ell m}^{*} a_{\ell^{\prime} m^{\prime}}\right\rangle=\delta_{\ell \ell^{\prime}} \delta_{m m^{\prime}} C_{\ell} .
$$

The variance $C_{\ell}$ is called the angular power of the multipole $\ell$. The higher $n$-point correlation functions are constrained in similar ways but, as we will see below, are not expected to provide independent information if a simple inflationary scenario was realized by nature.

2.2. Gaussianity. If inflation is driven by a single dynamically relevant degree of freedom with appropriate properties (minimal coupling, Minkowski vacuum in UV limit, etc.), then we can reduce the quantization of matter and spacetime fluctuations during inflation to the problem of quantizing free scalar fields. For free fields the only nontrivial object is the two-point correlation (the propagator), and all higher correlation functions either vanish or are just some trivial combination of the two-point function. This property is mapped onto the temperature field of the CMB. A classical random field with these properties is a Gaussian with mean $T_{0}$ and variance $C(\theta)$. Thus the brightness of the primordial CMB sky is completely characterized by $T_{0}$ and $C(\theta)$ (or $C_{\ell}$ ). Note that evolution of perturbations leads to deviations from Gaussianity that would mostly be evident at very small scales $(\ell \gg 100)$. Moreover, many inflationary models predict small deviations from Gaussianity; these are described in other contributions to this volume $[9,10]$.

2.3. Scale Invariance. Another generic feature of inflation is the almost scale invariance of the power spectrum of fluctuations. This can be understood easily, as the Hubble scale is approximately constant during inflation as the wavelengths of observable modes are redshifted beyond the horizon. Given that fluctuations of modes on horizon exit are related to the Hubble parameter, $\delta \phi=H / 2 \pi$, these modes have similar amplitudes. However, scale invariance is not exact. In canonical slow-roll inflation models, the deviation from exact scale invariance is due to the evolution of the Hubble parameter during inflation, which is measured by the so-called first slow-roll function $\epsilon_{1} \equiv \dot{d}_{\mathrm{H}}$ where $d_{\mathrm{H}} \equiv H^{-1}$ is the Hubble distance. From the weak energy condition $\epsilon_{1}>0$, while $\epsilon_{1} \ll 1$ during slow-roll inflation.

At the level of the angular power spectrum, exact scale invariance implies the Sachs-Wolfe "plateau" (i.e., constancy of $l(l+1) C_{\ell}$ at low $\left.\ell\right)[11]$

$$
C_{\ell}=\frac{2 \pi A}{\ell(\ell+1)}
$$

Here, again in the slow-roll parameterization, $A \sim$ $\left(H_{\text {infl }} / M_{\mathrm{P}}\right)^{2} T_{0}^{2} / \epsilon_{1}$. This neglects secondary anisotropies like the late time, integrated Sachs-Wolfe effect (particularly important at very low $\ell$ ) and the contribution from gravitational waves. Furthermore, inflation predicts a small departure from scale invariance, which has recently been detected (e.g., [6]), and which also contributes to a tilt in the aforementioned plateau.

2.4. Cosmic Variance. As we can measure only one sky, it is important to find the best estimators of $C_{\ell}$ and $C(\theta)$. Let us for the moment assume that we are able to measure the primordial CMB of the full-sky, without any instrumental noise. We also restrict ourselves to $\ell \geq 2$, as the variance of the monopole cannot be defined and the measured dipole is dominated by our motion through the universe rather than by primordial physics. (Separation of the Doppler dipole from the intrinsic dipole is possible in principle $[12,13]$, but not with existing data.) Statistical isotropy suggests to estimate the angular power by

$$
\hat{C}_{\ell}=\frac{1}{2 \ell+1} \sum_{m=-\ell}^{+\ell}\left|a_{\ell m}\right|^{2}
$$

which satisfies $\left\langle\hat{C}_{\ell}\right\rangle=C_{\ell}$ and is thus unbiased. The variance of this estimator can be calculated assuming Gaussianity:

$$
\operatorname{Var}\left(\hat{C}_{\ell}\right)=\frac{2}{2 \ell+1} \hat{C}_{\ell}^{2}
$$

It can be shown that, assuming statistical isotropy and Gaussianity, $\hat{C}_{\ell}$ is the best estimator in the sense that it has minimal variance and is unbiased. However, we emphasize that these qualities depend intrinsically on the correctness of the underlying assumptions. 
With these same assumptions, the variance of the twopoint correlation function is easily shown to be

$$
\operatorname{Var}[\hat{C}(\theta)]=\frac{1}{8 \pi^{2}} \sum_{\ell}(2 \ell+1) C_{\ell}^{2} P_{\ell}^{2}(\cos \theta),
$$

where $\hat{C}(\theta)$ is calculated from $\hat{C}_{\ell}$ following (1).

Putting the results of this section together allows us to come up with a generic prediction of inflationary cosmology for $C(\theta)$ on the largest angular scales; see Figure 2.

\section{Alignments}

In brief, the upshot of the previous section is that the twin assumptions of statistical isotropy and Gaussianity are the starting point of any $\mathrm{CMB}$ analysis. The measurements of the CMB monopole, dipole, and $(\Delta T)_{\mathrm{rms}}$ tell us that isotropy is observationally established at the percent level without any cosmological assumption, and at a level $10^{-4}$ if we attribute the dominant contribution to the dipole to our peculiar motion. For the purpose of cosmological parameter estimation, the task is to test the statistical isotropy of the CMB brightness fluctuations. At the largest angular scales, this can only be done by means of full-sky maps.

Let us assume that the various methods that have been developed to get rid of the Galactic foreground in single frequency band maps of the microwave sky are reliable (though we argue below that this might not be the case). Our review of alignments will be based on the internal linear combination (ILC) map produced by the WMAP team, which is based on a minimal variance combination of the WMAP frequency bands. The weights for the five frequency band maps are adjusted in 12 regions of the sky, one region lying outside the Milky Way and 11 regions along the Galactic plane.

3.1. Multipole Vectors. To study the orientation and alignment of $\mathrm{CMB}$ multipoles, Copi et al. [15] introduced to cosmology the use of multipole vectors; an alternative representation of data on the sphere. The multipole vectors contain information about the "directions" associated with each multipole. In this new basis the temperature fluctuation multipole, $\ell$, may be expanded as

$$
T_{\ell} \equiv \sum_{m=-\ell}^{\ell} a_{\ell m} Y_{\ell m} \equiv A^{(\ell)}\left[\prod_{i=1}^{\ell}\left(\hat{v}^{(\ell, i)} \cdot \hat{e}\right)-\mathcal{T}_{\ell}\right],
$$

where $\hat{v}^{(\ell, i)}$ is the $i$ th vector for the $\ell$ th multipole, $\hat{e}$ is the usual radial unit vector, $\mathcal{T}_{\ell}$ is the trace of the preceding product of multipole vector terms, and $A^{(\ell)}$ is the "power" in the multipole. By construction, we immediately see that $T_{\ell}$ is a symmetric traceless, rank $\ell$ tensor. Subtracting $\mathcal{T}_{\ell}$ ensures that $T_{\ell}$ is traceless and the dot products explicitly show this is a rotationally invariant quantity (a scalar under rotations). This form makes the symmetry properties obvious. As an example the quadrupole is written as

$$
T_{2}=A^{(2)}\left[\left(\hat{v}^{(2,1)} \cdot \hat{e}\right)\left(\hat{v}^{(2,2)} \cdot \hat{e}\right)-\frac{1}{3} \hat{v}^{(2,1)} \cdot \hat{v}^{(2,2)}\right] .
$$

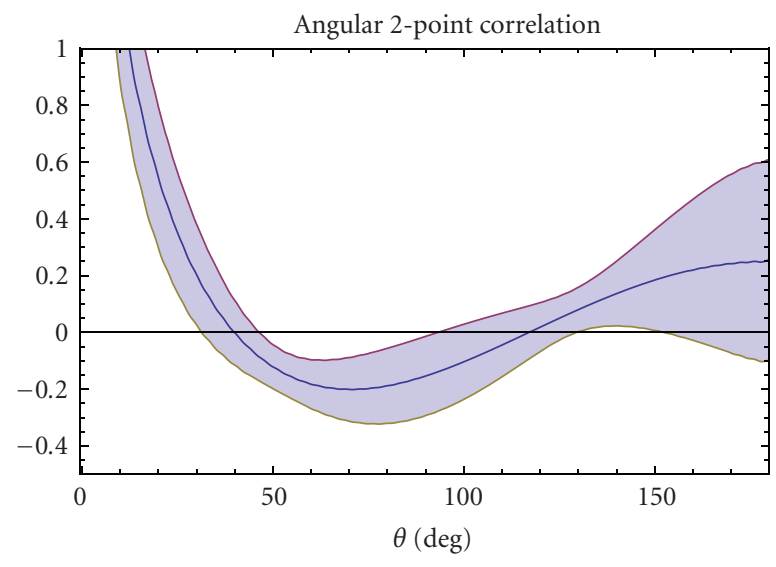

Figure 2: Mean and (cosmic) variance of the angular twopoint correlation function as expected from cosmological inflation (arbitrary normalization). Only statistical isotropy, Gaussianity and scale invariance are assumed. Tensors, spectral tilt, reionization and the integrated Sachs-Wolfe effect are neglected for the purpose of this plot. Comparison to the prediction from the best-fit $\Lambda \mathrm{CDM}$ model (Figure 5) reveals that these corrections are subdominant. Note that cosmic variance errors at different values of $\theta$ are very highly correlated.

These two forms for representing $T_{\ell}$, harmonic, and multipole-vector, both contain the same information. At the same time, they are fundamentally different from each other. Each unit vector $\hat{v}^{(\ell, i)}$ has two degrees of freedom while the scalar, $A^{(\ell)}$ has one; thus the multipole vector representation contains the full $2 \ell+1$ degrees of freedom. Note that we call each $\hat{v}^{(\ell, i)}$ a multipole vector but it is only defined up to a sign. We can always reflect the vector through the origin by absorbing a negative sign into the scalar $A^{(\ell)}$; thus these vectors actually are headless. Regardless, we will continue to refer to them as multipole vectors and not use the overall sign of the vector in our analysis. This issue is equivalent to choosing a phase convention, such as the Condon-Shortley phase for the spherical harmonics. For the work reviewed here the overall phase is not relevant and thus will not be specified.

An efficient algorithm to compute the multipole vectors for low- $\ell$ has been presented in [15] and is publicly available [16]; other algorithms have been proposed as well in [1719]. Interestingly, after the publication of the CHS paper [15], Weeks [18] pointed out that multipole vectors were actually first used by Maxwell [20] more than 100 years ago in his study of multipole moments in electrodynamics. They remain in use in geometrology, nuclear physics, and other fields.

The relation between multipole vectors and the usual harmonic basis is very much the same as that between Cartesian and spherical coordinates of standard geometry: both are complete bases, but specific problems are much more easily addressed in one basis than the other. In particular, we and others have found that multipole vectors are particularly well suited for tests of planarity and alignment of the CMB anisotropy pattern. Moreover, a number of 


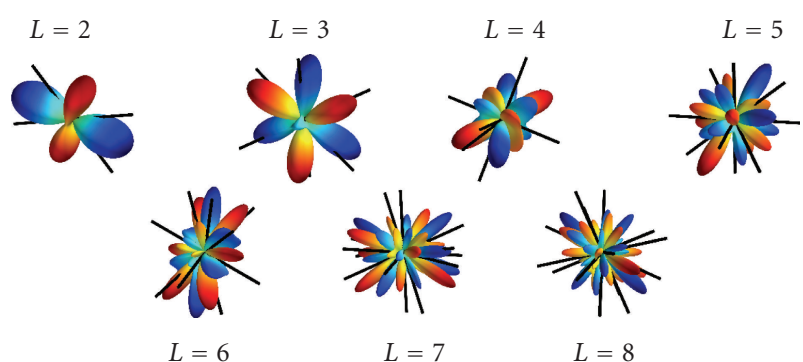

FIGURE 3: Multipole vectors of our sky, based on WMAP five-year full-sky ILC map and with galactic plane coinciding with the plane of the page. The temperature pattern at each multipole $\ell(2 \leq \ell \leq 8)$ can either be described by an angular temperature pattern (colored lobes in this figure), or alternatively by precisely $\ell$ multipole vectors (black "sticks"). While the multipole vectors contain all information about the directionality of the CMB temperature pattern, they are not simply related to the hot and cold spots and, for example, do not correspond to the temperature minima/maxima [14]. Notice that $\ell=2$ and 3 temperature patterns are rather planar with the same plane and that their vectors lie approximately in this plane. Adopted from [15].

interesting theoretical results have been found; for example, Dennis and Phys [21] analytically computed the two-point correlation function of multipole vectors for a Gaussian random, isotropic underlying field. Numerous quantities have been proposed for assigning directions to multipoles and statistics on these quantities have been studied. In the work of Copi et al. [14] we have summarized these attempts and have shown their connections to the multipole vectors.

3.2. Planarity and Alignments. Tegmark et al. [22] and de Oliveira-Costa et al. [23] first argued that the octopole is planar and that the quadrupole and octopole planes are aligned. In the work of Schwarz et al. [24], followed up by Copi et al. $[14,25]$, we investigated the quadrupoleoctopole shape and orientation using the multipole vectors. The quadrupole is fully described by two multipole vectors, which define a plane. This plane can be described by the "oriented area" vector

$$
\vec{w}^{(\ell ; i, j)} \equiv \widehat{v}^{(\ell, i)} \times \widehat{v}^{(\ell, j)} .
$$

(Note that the oriented area vector does not fully characterize the quadrupole, as pairs of quadrupole multipole vectors related by a rotation about the oriented area vector lead to the same oriented area vector.) The octopole is defined by three multipole vectors which determine (but again are not fully determined by) three area vectors. Hence there are a total of four planes determined by the quadrupole and octopole.

In the work of Copi et al. [25] we found that (see Figure 4)

(i) the four area vectors of the quadrupole and octopole are mutually close (i.e., the quadrupole and octopole planes are aligned) at the $99.6 \%$ C.L.;

(ii) the quadrupole and octopole planes are orthogonal to the ecliptic at the $95.9 \%$ C.L.; this alignment was at

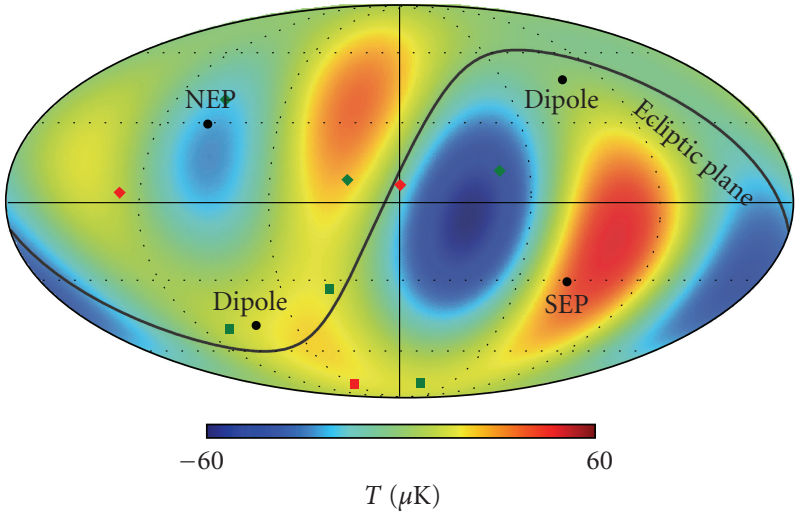

FIGURE 4: Quadrupole and octopole $(\ell=2$ and 3$)$ temperature anisotropy of the WMAP sky map in galactic coordinates, shown with the ecliptic plane and the cosmological dipole. Included are the multipole vectors (solid diamonds): two for the quadrupole (red diamonds) and three for the octopole (green diamonds). We also show the four normals (solid squares) to the planes defined by vectors that describe the quadrupole and octopole temperature anisotropy; one normal is defined by the quadrupole (red square) and three by the octopole (green squares). Note that three out of four normals lie very close to the dipole direction. The probability of this alignment being accidental is about one part in a thousand. Moreover, the ecliptic plane traces out a locus of zero of the combined quadrupole and octopole over a broad swath of the skyneatly separating a hot spot in the northern sky from a cold spot in the south. These apparent correlations with the solar system geometry are puzzling and currently unexplained.

98.5\% C.L. in our analysis of the WMAP 1 year maps. The reduction of alignment was due to WMAP's adaption of a new radiometer gain model for the 3 year data analysis, that took seasonal variations of the receiver box temperature into account-a systematic that is indeed correlated with the ecliptic plane. We regard that as clear evidence that multipole vectors are a sensitive probe of alignments;

(iii) the normals to these four planes are aligned with the direction of the cosmological dipole (and with the equinoxes) at a level inconsistent with Gaussian random, statistically isotropic skies at 99.7\% C.L.;

(iv) the ecliptic threads between a hot and a cold spot of the combined quadrupole and octopole map, following a node line across about $1 / 3$ of the sky and separating the three strong extrema from the three weak extrema of the map; this is unlikely at about the 95\% C.L.

These numbers refer to the WMAP ILC map from three years of data; other maps give similar results. Moreover, correction for the kinematic quadrupole-slight modification of the quadrupole due to our motion through the $\mathrm{CMB}$ rest frame-must be made and increases significance of the alignments. See [25, Table 3] for the illustration of both of these points.

While not all of these alignments are statistically independent, their combined statistical significance is certainly 
greater than their individual significances; for example, given their mutual alignments, the conditional probability of the four normals lying so close to the ecliptic is less than $2 \%$; the combined probability of the four normals being both so aligned with each other and so close to the ecliptic is less than $0.4 \% \times 2 \%=0.008 \%$. These are therefore clearly surprising, highly statistically significant anomalies - unexpected in the standard inflationary theory and the accepted cosmological model.

Particularly puzzling are the alignments with solar system features. $\mathrm{CMB}$ anisotropy should clearly not be correlated with our local habitat. While the observed correlations seem to hint that there is contamination by a foreground or perhaps by the scanning strategy of the telescope, closer inspection reveals that there is no obvious way to explain the observed correlations. Moreover, if their explanation is that they are a foreground, then that will likely exacerbate other anomalies that we will discuss in Section 4.2.

Our studies (see [14]) indicate that the observed alignments are with the ecliptic plane, with the equinox, or with the $\mathrm{CMB}$ dipole, and not with the Galactic plane: the alignments of the quadrupole and octopole planes with the equinox/ecliptic/dipole directions are much more significant than those for the Galactic plane. Moreover, it is remarkably curious that it is precisely the ecliptic alignment that has been found on somewhat smaller scales using the power spectrum analyses of statistical isotropy [26-29].

Finally, it is important to make sure that the results are unbiased by unfairly chosen statistics. We have studied this issue extensively in [14], and here we briefly describe the principal statistics used to quantify the probability of alignments quoted just above.

To define statistics we first compute the three dotproducts between the quadrupole area vector and the three octopole area vectors:

$$
A_{k} \equiv\left|\vec{w}^{(2 ; 1,2)} \cdot \vec{w}^{(3 ; i, j)}\right| .
$$

The absolute value is included since the multipole vectors are headless; thus each $A_{k}$ lies in the interval $[0,1]$. Two natural choices of statistics that are independent of the ordering of $A_{k}$ are

$$
\begin{gathered}
S \equiv \frac{1}{3}\left(A_{1}+A_{2}+A_{3}\right), \\
T \equiv 1-\frac{1}{3}\left[\left(1-A_{1}\right)^{2}+\left(1-A_{2}\right)^{2}+\left(1-A_{3}\right)^{2}\right] .
\end{gathered}
$$

Both $S$ and $T$ statistics can be viewed as the suitably defined "distance" to the vertex $\left(A_{1}, A_{2}, A_{3}\right)=(0,0,0)$. A third obvious choice, $\left(A_{1}^{2}+A_{2}^{2}+A_{3}^{2}\right) / 3$, is just $2 S-T$. To test alignment of the quadrupole and octopole planes with one another we quoted the $S$ statistic numbers; $T$ gives similar results.

Alternatively, generalizing the definition in [22], one can find, for each $\ell$, the choice, $n_{\ell}$, of $z$ axis that maximizes the angular momentum dispersion

$$
\widehat{L}_{\ell}^{2} \equiv \frac{\sum_{m=-\ell}^{\ell} m^{2}\left|a_{\ell m}\right|^{2}}{\ell^{2} \sum_{m=-\ell}^{\ell}\left|a_{\ell m}\right|^{2}} .
$$

One can then compare the maximized value with that from simulated isotropic skies [14]. Because $\ell=2,3$ are both planar (the quadrupole trivially so, the octopole because the three planes of the octopole are nearly parallel), the direction that maximizes the angular momentum dispersion of each is nearly the same as the (average) direction of that multipole's planes. Thus, the alignment of the octopole and quadrupole can be seen either from the $s$ statistic, or by looking at the alignment of $n_{2}$ with $n_{3}$.

To test alignments of multipole planes with physical directions, we find the plane whose normal, $\hat{n}$, has the largest dot product with the sum of the four quadrupole and octopole area vectors [14]. Again, since $\vec{w}_{i} \cdot \hat{n}$ is defined only up to a sign, we take the absolute value of each dot product. Therefore, we find the direction $\hat{n}$ that maximizes

$$
\& \equiv \frac{1}{N_{\ell}} \sum_{i=1}^{N_{\ell}}\left|\vec{w}_{i} \cdot \hat{n}\right| .
$$

3.3. Summary. The study of alignments in the low $\ell$ CMB has found a number of peculiarities. We have shown that the alignment of the quadrupole and octopole planes is inconsistent with Gaussian, statistically isotropic skies at least at the $99 \%$ confidence level. Further a, number of (possibly related) alignments occur at 95\% confidence levels or greater. Putting together these provides a strong indication that the full-sky CMB WMAP maps are inconsistent with the standard cosmological model at the large-angles. Even more peculiar is the alignment of the quadrupole and octopole with solar system features (the ecliptic plane and the dipole).

This is strongly suggestive of an unknown systematic in the data reduction; however, careful scrutiny has revealed no such systematic (except the mentioned modification of the radiometer gain model, that leads to a reduction of ecliptic alignment); see Sections 5.3 and 5.4 for further discussion of the data analysis and instrumental explanations. We again stress that these results hold for full-sky maps; maps that are produced through combination of the individual frequency maps in such a way as to remove foregrounds. An alternative approach that removes the need for full-sky maps is presented in the next section.

\section{Two-point Angular Correlation Function}

The usual CMB analysis solely involves the spherical harmonic decomposition and the two-point angular power spectrum. There are many reasons for this. Firstly, when working with a statistically isotropic universe the angular power spectrum contains all of the physical information. Secondly, the standard theory predicts the $a_{\ell m}$ and their statistical properties, through the $C_{\ell}$, thus the spherical harmonic basis is a natural one to employ. Finally, as measured today the angular size of the horizon at the time of last scattering is approximately 1 degree. Since $\theta$ (deg)200/ $\ell$, the causal physics at the surface of last scattering leaves its imprint on the $\mathrm{CMB}$ on small scales, $\theta \lesssim 1^{\circ}$ or $\ell \gtrsim 100$. The two-point angular power spectrum focuses on these small scales, making it a good means of exploring the physics of 
the last scattering surface. The tremendous success of the standard model of cosmology has been the agreement of the theory and observations on these small scales, allowing for the precise determination of the fundamental cosmological parameters [30].

The two-point angular correlation function provides another means of analyzing CMB observations and should not be ignored even if, in principle, it contains the same information as the angular power spectrum. Thus, even in the case of full-sky observations and/or statistical isotropy there are benefits in looking at the data in different ways. The situation is similar to a function in one dimension where it is widely appreciated that features easily found in the real space analysis can be very difficult to find in the Fourier transform, and vice versa. Furthermore, the twopoint angular correlation function highlights behavior at large-angles (small $\ell$ ); the opposite of the two-point angular power spectrum. Thus the angular correlation function allows for easier study of the temperature fluctuation modes that are super-horizon sized at the time of last scattering. Finally, the angular correlation function in its simplest form is a direct pixel-based measure (see below). Thus it does not rely on the reconstruction of contaminated regions of the sky to employ. This makes it a simple, robust measure even for partial sky coverage.

4.1. Definition. Care should be taken when discussing statistical quantities of the $\mathrm{CMB}$ and their estimators. It rarely is in the literature. Here we follow the notation of Copi et al. [25], also see [31]. The two-point angular correlation function,

$$
\widetilde{\mathcal{C}}\left(\hat{e}_{1}, \hat{e}_{2}\right) \equiv\left\langle T\left(\hat{e}_{1}\right) T\left(\hat{e}_{2}\right)\right\rangle,
$$

is the ensemble average (represented by the angle brackets, $\langle\cdot\rangle)$ of the product of the temperatures in the directions $\hat{e}_{1}$ and $\hat{e}_{2}$. Unfortunately we only have one universe to observe so this ensemble average cannot be calculated. Instead we average over the sky so that what we mean by the two-point angular correlation function is a sky average,

$$
\mathcal{C}(\theta) \equiv \overline{T\left(\hat{e}_{1}\right) T\left(\hat{e}_{2}\right)},
$$

where the average is over all pairs of pixels with $\hat{e}_{1} \cdot \hat{e}_{2}=$ $\cos \theta$. This is a pixel-based quantity and can be calculated for any region of the sky (of course not all separations $\theta$ may be represented on a given patch of the sky, depending on its geometry).

4.2. Missing Angular Power at Large Scales. Spergel et al. [2] found that the two-point correlation function nearly vanishes on scales greater than about 60 degrees, contrary to what the standard $\Lambda \mathrm{CDM}$ theory predicts, and in agreement with the same finding obtained from COBE data about a decade earlier [32].

We have revisited the angular two-point function in the 3-yr WMAP data in [25] and the 5-yr WMAP data in [31]; see Figure 5. From this figure we qualitatively see the following.

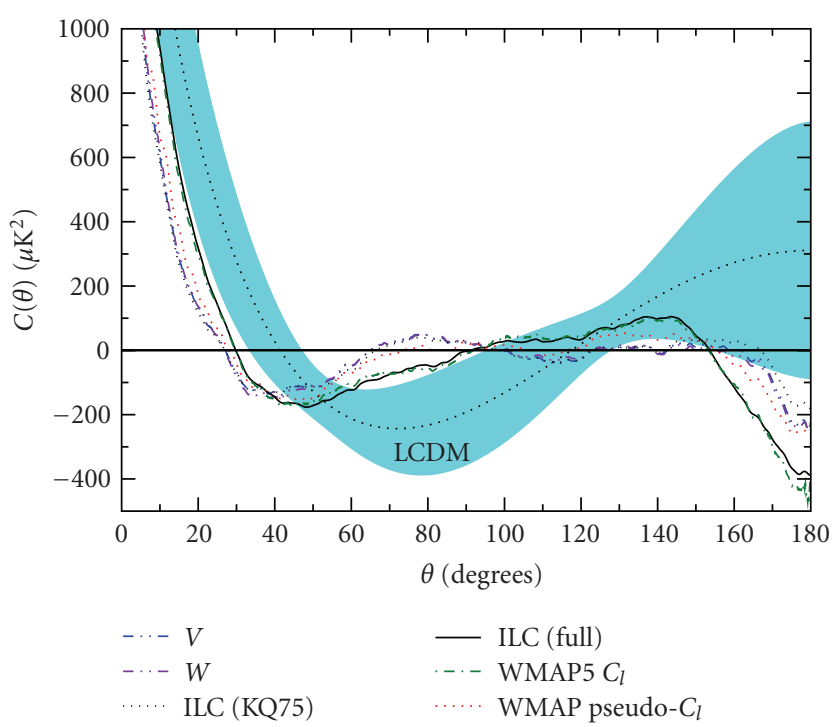

FIgure 5: Two-point angular correlation function, $\mathcal{C}(\theta)$, computed in pixel space, for three different bands masked with the KQ75 mask (from WMAP 5 year data). Also shown are the correlation function for the ILC map with and without the mask, and the value expected for a statistically isotropic sky with best-fit $\Lambda$ CDM cosmology together with $68 \%$ cosmic variance error bars. Even by eye, it is apparent that masked maps have $C(\theta)$ that is consistent with zero at $\theta \gtrsim 60 \mathrm{deg}$. We also show the $C(\theta)$ computed from the "official" published maximum likelihood estimator-based $C_{\ell}$. Clearly, the MLE-based $C_{\ell}$, as well as $\mathcal{C}(\theta)$ computed from the full-sky ILC maps, is in significant disagreement with the angular correlation function computed from cut-sky maps. Adopted from [31].

(i) All of the cut-sky map curves are very similar to each other, and they are also very similar to the Legendre transform of the pseudo- $C_{\ell}$ estimate of the angular power spectrum, which is not surprising given that the two are formally equivalent [33]. Meanwhile the full-sky ILC $\mathcal{C}(\theta)$ and the Legendre transform of the maximum likelihood estimator (MLE) of the $C_{\ell}$ agree well with each other, but not with any of the others.

(ii) The most striking feature of the cut-sky (and pseudo$\left.C_{\ell}\right) \mathcal{C}(\theta)$, is that all of them are very nearly zero above about $60^{\circ}$, except for some anticorrelation near $180^{\circ}$. This is also true for the full-sky curves, but less so.

In order to be more quantitative about these observations we adopt the $S_{1 / 2}$ statistic introduced by the WMAP team [2] which quantifies the deviation of the two-point correlation function from zero:

$$
S_{1 / 2} \equiv \int_{-1}^{1 / 2}[\mathcal{C}(\theta)]^{2} d(\cos \theta) .
$$

Spergel et al. [2] found that only $0.15 \%$ of the parameter sets in their Markov chain of $\Lambda \mathrm{CDM}$ model CMB skies had lower values of $S_{1 / 2}$ than the observed one-year WMAP sky.

Applying this statistic we have found that the two-point function computed from the various cut-sky maps shows an even stronger lack of power, for WMAP 5 year maps 
significant at the $0.037 \%-0.025 \%$ level depending on the map used; see Figure (5). However, we also found that, while $\mathcal{C}(\theta)$ computed in pixel space over the masked sky agrees with the harmonic space calculation that uses the pseudo- $C_{\ell}$ estimator, it disagrees with the $C_{\ell}$ obtained using the MLE (advocated in the 3rd year WMAP release [4]). The MLEbased $C_{\ell}$ leads to $C(\theta)$ that is low (according to the $S_{1 / 2}$ statistic) only at the $4.6 \%$ level.

There are actually two interesting questions one can ask.

(i) Is the correlation function measured on the cutsky compatible with cut-sky expectation from the Gaussian random, isotropic underlying model?

(ii) Is the reconstruction of the full-sky correlation function from partial information compatible with the expectation from the Gaussian random, isotropic underlying model?

Our results refer to the first question above. The second question, while also extremely interesting, is more difficult to be robustly resolved because the reconstruction uses assumptions about statistical isotropy (see the next subsection).

The little large-angle correlation that does appear in the full-sky maps (e.g., the solid, black line in Figure 5) is associated with points inside the masked region. Shown in Figure 6 are the normalized contributions to $\mathcal{C}(\theta)$ from different parts of the map. In particular, we see that almost all of the contribution to the full-sky two-point angular correlation function comes from correlations with at least one point inside the masked region. Conversely, there is essentially no large-angle correlation for points outside the masked region and even very little among the points completely inside the mask. We also see that all the curves cross zero at nearly the same angle, $\theta \sim 90^{\circ}$. We have no explanation for these results though they may point to systematics in the data.

4.3. Alternative Statistics. The two-point angular correlation function, $\mathcal{C}(\theta)$, as defined above in (15) is a simple pixelbased measure of correlations. It makes no assumptions about the underlying theory, which can be taken as a feature or as a flaw. On the positive side, (15) does not assume that the standard model is correct and trys to "force" it on the data. On the negative side we are not utilizing the full information available when comparing to the standard model.

Various approaches have been taken to incorporate the standard model in the analysis, for example; Hajian [34] defined a statistic that explicitly takes into account the covariance in the quantity $C(\theta)$ :

$$
A(x) \equiv \int_{-1}^{x} \int_{-1}^{x} C(\theta) F^{-1}\left(\theta, \theta^{\prime}\right) C\left(\theta^{\prime}\right) d(\cos \theta) d\left(\cos \theta^{\prime}\right),
$$

where $F$ is the aforementioned covariance

$$
F\left(\theta, \theta^{\prime}\right) \equiv\left\langle[C(\theta)-\langle C(\theta)\rangle]\left[C\left(\theta^{\prime}\right)-\left\langle C\left(\theta^{\prime}\right)\right\rangle\right]\right\rangle,
$$

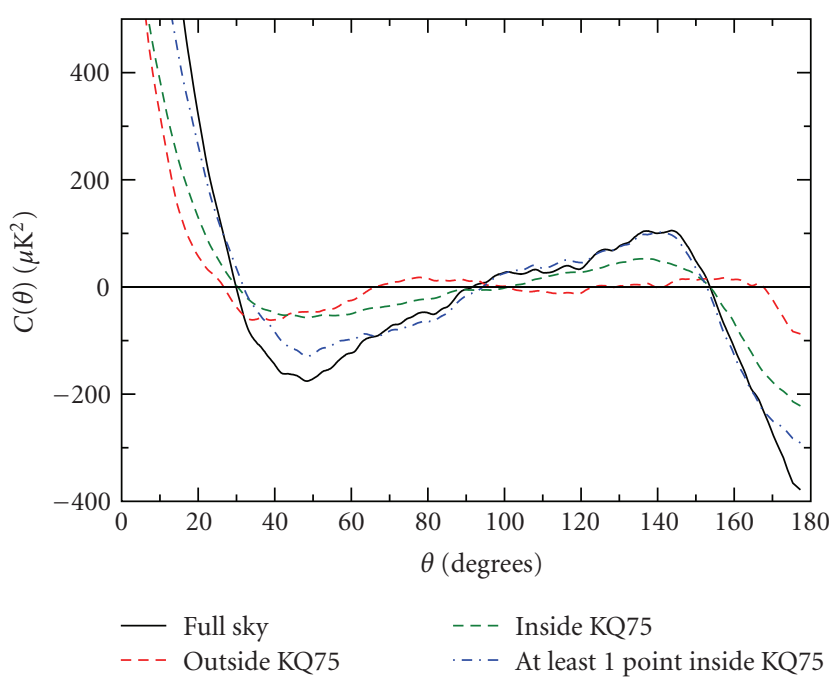

Figure 6: The two-point angular correlation function from the WMAP 5-year results. Plotted are $\mathcal{C}(\theta)$ for the ILC calculated separately on the part of the sky outside the KQ75 cut (dashed line), inside the KQ75 cut (dotted line), and on the part of the sky with at least on point inside the KQ75 cut (dotted-dashed line). For better comparison to the full-sky $\mathcal{C}(\theta)$ (solid line), the partial sky $\mathcal{C}(\theta)$ has been scaled by the fraction of the sky over which they are calculated. Adopted from [31].

and as usual the angle brackets denote an ensemble average. Note that in the limit that $C(\theta)$ is uncorrelated then $A(1 / 2)=$ $S_{1 / 2}$. Clearly this statistic relies on a model to calculate $F\left(\theta, \theta^{\prime}\right)$. With this statistic and assuming the concordance model; it is found that less than $1 \%$ of realizations of the standard model have au $A(0.53)$ less than those found for the masked skies. For the full-sky ILC map approximately $8 \%$ of realizations have a smaller value. Though less constraining than making no assumptions about the theory through the use of the $S_{1 / 2}$ statistic, these results are consistent with those we previously found.

Another approach advocated by Efstathiou et al. [35] is to reconstruct the full-sky $C(\theta)$ from the partial sky and compare the reconstructed full-sky $C(\theta)$ (using, say $S_{1 / 2}$ ) to the predictions of the model. This approach employs the usual map making algorithm on the low- $\ell$ spherical harmonic coefficients, $a_{\ell m}$. In this approach it is assumed that the statistical properties of the $a_{\ell m}$ above some $\ell_{\max }$ are known. In particular it assumes there are no correlations between the $a_{\ell m}$ with $\ell<\ell_{\max }$ and those with $\ell>\ell_{\max }$. The method is very similar to a maximum likelihood analysis (see e.g., [36]). As we have seen above (e.g., in Figure 5) it is not surprising that this approach will be consistent with the fullsky ILC results, as these authors have verified. As mentioned in the previous subsection, however, this procedure poses a different question of the data than the one that has been addressed by the $S_{1 / 2}$ statistic applied to a masked sky. With the map-making technique a full-sky map is constructed that is consistent with the sky outside the mask but relies on assumptions to fill in the masked region. As is clear from Figure 6 it is precisely the region inside the mask that is 
introducing correlations with the region outside the mask. Thus, the assumptions required to allow filling in the masked region also produce two-point angular correlations.

Whether or not reconstructing the full-sky is a "more optimal" approach than direct calculation of the cut-sky $C(\theta)$ is moot, but likely depends on the actual (rather than the assumed) statistical properties of the underlying fluctuations as well as on the particular realization of those distributions. What is important is to make an "apples to apples" comparison between the observed sky and simulated realizations of the ensemble of possible skies. As we have shown, the pixel-based two-point correlation function on the region of the sky outside a conservative galactic mask is inconsistent with the predictions of the standard $\Lambda \mathrm{CDM}$ model for the identical pixel-based two-point correlation function on the identically masked sky. The fact that the full-sky analysis shows less statistical significance is not in contradiction with the cut-sky result, although it may eventually help in pointing to a cause of this anomaly.

4.4. Summary. The striking feature of the two-point angular correlation function as seen in Figure 5 is not that it disagrees with $\Lambda$ CDM (though it does at $>90 \%$ C.L.) but that at large-angles it is nearly zero. This lack of large-angle correlations is unexpected in inflationary models. The $S_{1 / 2}$ statistic quantifies the deviation from zero and shows that a discrepancy exists at more than $99.9 \%$ C.L. Equally striking is the fact that the little correlation that does exist in the full-sky ILC map, or equally in the MLE estimated $a_{\ell m}$, comes from correlations between the masked foreground region and the expectedly cleaner CMB regions of the sky. Thus this residual correlation, which still is discrepant with generic inflationary predictions at about $95 \%$ C.L., comes from the reconstruction procedure. This surprising lack of large-angle correlation outside the masked region remains an open problem.

We also note that the vanishing of power is much more apparent in real space (as in $\mathcal{C}(\theta)$ ) than in multipole space (as in $C_{\ell}$ ). The harmonic-space quadrupole and octopole are only moderately low (e.g., [37]), and it is really a range of low multipoles that conspires to make up the vanishing $\mathcal{C}(\theta)$. Specifically, as discussed in [31], there is a cancellation between the combined contributions of $C_{2}, \ldots, C_{5}$ and the contributions of $C_{\ell}$ with $\ell \geq 6$. It is this conspiracy that is most disturbing, since it violates the independence of the $C_{\ell}$ of different $\ell$ that defines statistical isotropy.

In [31] we therefore explored the possibility that the vanishing of $C(\theta)$ could be explained simply by changing the values of the theoretical low- $\ell C_{\ell}$, as might be the result, say, of a modified inflaton potential. In particular, we replaced $C_{2}$ through $C_{20}$ in the best-fit $\Lambda \mathrm{CDM}$ model with the values extracted from the cut-sky ILC five-year map. From these $C_{\ell}$ 's, 200000 random maps were created, masked, and $S_{1 / 2}$ computed. Under the assumptions of Gaussianity and statistical isotropy of these $C_{\ell}$ 's only 3 percent of the generated maps had $S_{1 / 2}$ less than the cut-sky ILC5 value. Thus, even if the $C_{\ell}$ are set to the specific values that produce such a low $S_{1 / 2}$, a Gaussian random, statistically isotropic realization is unlikely to produce the observed lack of largeangle correlations at the $97 \%$ C.L. Moreover, in work in progress we show that almost all of those $3 \%$ are skies with several anomalously low $C_{\ell}$-not at all the sky we see. Only a tiny fraction of the $3 \%$ represent skies in which most of the individual $C_{\ell}$ 's were close to the $\Lambda \mathrm{CDM}$ prediction but they conspire to cancel one another in the large-angle $C(\theta)$. This shows that either (i) the low- $\ell C_{\ell}$ 's are correlated, contrary to the assumption of statistical isotropy or (ii) our Universe is an extremely unlikely realization of whatever statistically isotropic model one devises (Though this appears to be an unlikely explanation since correlations between the $C_{\ell}$ generically increase the variance of the $S_{1 / 2}$ statistic [33]).

It is for this reason that theoretical efforts to explain "low power on large scales" must focus on explaining the low $\mathcal{C}(\theta)$ at $\theta \gtrsim 60 \mathrm{deg}$, rather than the low quadrupole.

Finally, one might ask if the observed lack of correlation and the alignment of quadrupole and octopole are correlated. This issue was studied by Rakić \& Schwarz [38] for the full-sky and by Sarkar et al. [39] for the cut-sky case. In both cases, it was shown that low power and alignments are uncorrelated, that is, that having one does not imply a larger or smaller probability of having the other. This was shown by applying a Monte-Carlo analysis to sky realizations with the underlying standard Gaussian random, statistically isotropic cosmological model, without any further constraints. Thus one might view the $99.6 \%$ C.L. of quadrupole-octopole alignment presented in the previous section and the 95\% C.L. for lack of correlation in full-sky maps reported in this section as statistically independent.

\section{Quest for an Explanation}

Understanding the origin of $\mathrm{CMB}$ anomalies is clearly important. Both the observed alignments of the low- $\ell$ fullsky multipoles, and the absence of large-angle correlations (especially on the galaxy cut-sky) are severely inconsistent with predictions of standard cosmological theory. There are four classes of possible explanations:

(1) astrophysical foregrounds,

(2) artifacts of faulty data analysis,

(3) instrumental systematics,

(4) theoretical/cosmological.

In this section, we review these four classes of explanations, giving examples from each. First, however, we discuss two generic ways to break statistical isotropy and affect the intrinsic (true) CMB signal-additive and multiplicative modulations-and illustrate in general terms why it has been so difficult to explain the anomalies.

5.1. Additive versus Multiplicative Effects. Why is it difficult to explaining the observed $\mathrm{CMB}$ anomalies? There are three basic reasons:

(i) Most explanations work by adding power to the largeangle $\mathrm{CMB}$, while the observed anisotropies actually 
have less large-scale power and particularly less largeangle correlation, than the $\Lambda \mathrm{CDM}$ cosmological model predicts.

(ii) Unaccounting for sources of CMB fluctuations in the foreground, even if possessing/causing aligned low$\ell$ multipoles of their own, cannot bring unaligned statistically isotropic cosmological perturbations into alignment. Therefore, aligned foregrounds as an explanation for alignment work only if the cosmological signal is subdominant, thus exacerbating the lack of large-angle correlations.

(iii) The alignments of the quadrupole and octopole are with respect to the ecliptic plane and near the dipole direction. It is generally difficult to have these directions naturally be picked out by any class of explanations (though there are exceptions to thissee the instrumental example below).

Gordon et al. [40], Rakić and Schwarz [38] and Bunn and Bourdon [41] explored generic "additive models" where the temperature modification that causes the alignment is added to

$$
T_{\text {observed }}(\hat{e})=T_{\text {intrinsic }}(\hat{e})+T_{\text {add }}(\hat{e}) .
$$

Here $T_{\text {add }}(\hat{e})$ is the additive term-perhaps contamination by a foreground and perhaps an additive instrumental or cosmological effect. They showed that additive modulations of the CMB sky that ameliorate the alignment problems tend to worsen the overall likelihood at large scales (though they may pick up offsetting positive likelihood contribution from higher multipoles). The intuitive reason for this is that there are two penalties incurred by the additive modulation. First, since the power spectrum at low $\ell$ is lower than expected, one typically needs to arrange for an accidental cancellation between $T_{\text {intrinsic }}$ and $T_{\text {add }}$; the cancellation moreover must leave aligned quadrupole and octopole even though the quadrupole and octopole of $T_{\text {intrinsic }}$ are not aligned. (Very similar reasoning argues against additive explanations of the suppression of large-angle correlations.) Second, the simplest models for the additive contribution that are based on an azimuthally symmetric modulation of a gradient field can only affect $m=0$ multipoles around the preferred axis, while as we mentioned earlier the observed quadrupole and octopole as seen in the preferred (dipole) frame are dominated by the $m=\ell$ components.

In contrast to the additive models, the multiplicative mechanisms, where the intrinsic temperature is multiplied by a spatially varying modulation, are phenomenologically more promising. As a proof of principle, a toy-model modulation [40]

$$
T_{\text {observed }}(\widehat{e})=f\left[1+w_{2} Y_{20}(\widehat{e})\right] T_{\text {intrinsic }}(\widehat{e})
$$

(where the modulation is a pure $Y_{20}$ along the dipole axis) can increase the likelihood of the WMAP data by a factor of $\exp (16 / 2)$ and, at the same time, increase the probability of obtaining a sky with more alignment (e.g., higher angular momentum statistic) 200 times, to $45 \%$; see Figure 7.
Indeed, Groeneboom et al. [42], building on the work of Groeneboom and Eriksen [43] and Hanson and Lewis [44] and motivated by a model due to Ackerman et al. [45], have identified a $9 \sigma$ quadrupolar power asymmetry, recently confirmed by the WMAP team [46]; this anomaly can, however, be fully explained by accounting for asymmetric beams [47]. Recently, Hoftuft et al. [48] found a greater than $3-\sigma$ evidence for nonzero dipolar modulation of the power.

5.2. Astrophysical Explanations. One fairly obvious possibility is that there is a pernicious foreground that contaminates the primordial $\mathrm{CMB}$ and leads to the observed anomalies. Such foregrounds are, of course, additive mechanisms, in the sense of the preceding section, and so suffer from the shortcomings described therein. Moreover, most such foregrounds are Galactic, while the observed alignments are with respect to the ecliptic plane. One would expect that Galactic foregrounds should lead to Galactic and not ecliptic foregrounds. This simple expectation was confirmed in [14], where we showed that, by artificially adding a large admixture of Galactic foregrounds to WMAP CMB maps, the quadrupole vectors move near the $z$-axis and the normal into the Galactic plan, while for the octopole all three normals become close to the Galactic disk at $90^{\circ}$ from the Galactic center. Therefore, as expected Galactic foregrounds lead to Galactic, and not ecliptic, correlations of the quadrupole and octopole (see also studies by $[49,50]$ ).

Moreover, in [14], we have shown that the known Galactic foregrounds possess a multipole vector structure very different from that of the observed quadrupole and octopole. The quadrupole is nearly pure $Y_{22}$ in the frame where the $z$-axis is parallel to the dipole (or $\hat{w}^{(2,1,2)}$ or any nearly equivalent direction), while the octopole is dominantly $Y_{33}$ in the same frame. Mechanisms which produce an alteration of the microwave signal from a relatively small patch of skyand all of the recent proposals fall into this class-are most likely to produce aligned $Y_{20}$ and $Y_{30}$. This is essentially because the low- $\ell$ multipole vectors will all be parallel to each other, leading to a $Y_{\ell 0}$ in this frame.

A number of authors have attempted to explain the observed quadrupole-octopole correlations in terms of a new foreground-for example the Rees-Sciama effect [38, 51], interstellar dust [52], local voids [53], or the SunyaevZeldovich effect [54]. Most if not all of these proposals have a difficult time explaining the anomalies without severe finetuning. For example, Vale [55] cleverly suggested that the moving lens effect, with the Great Attractor as a source, might be responsible for the extra anisotropy; however, Cooray and Seto [56] have argued that the lensing effect is far too small and requires too large a mass of the Attractor.

It is also interesting to ask if any known or unknown Solar system physics could lead to the observed alignments. Dikarev et al. [57, 58] studied the question of whether solar system dust could give rise to sizable levels of microwave emission or absorption. Surprisingly, very little is known about dust grains of $\mathrm{mm}$ to $\mathrm{cm}$ size in the Solar system, and their absorption/emission properties strongly depend on their chemical composition. While iron and ice particles 


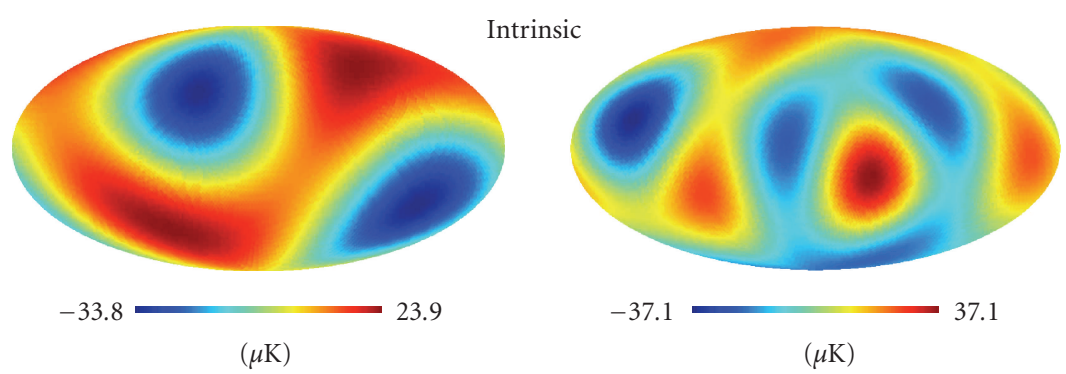

(a)

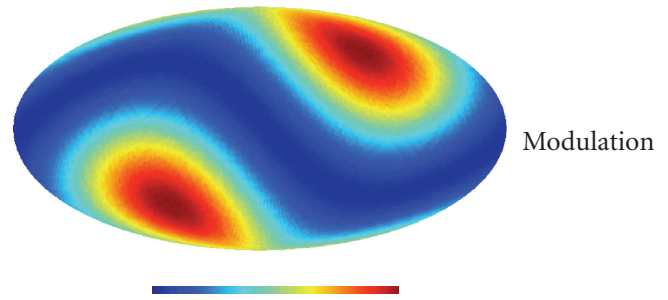

(b)

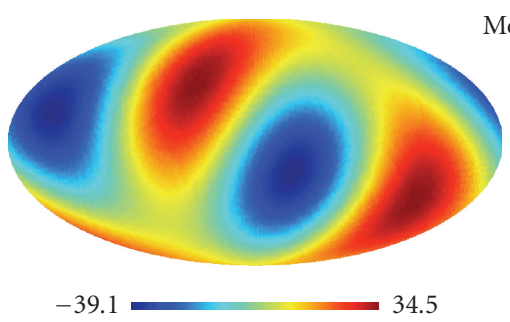

$(\mu \mathrm{K})$

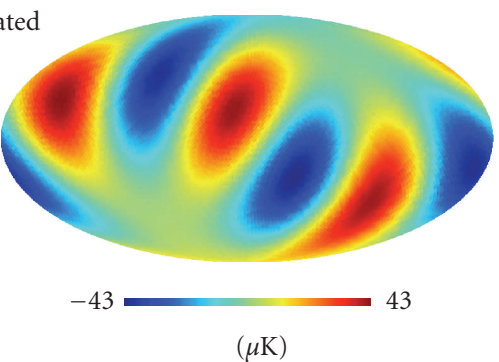

(c)
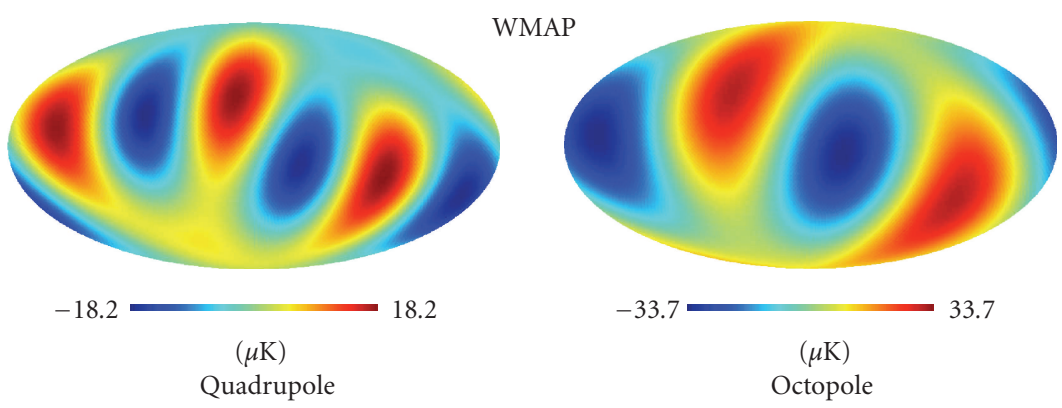

(d)

FIGURE 7: A realization of the multiplicative model where the quadrupole (left column) and octopole (right column) exhibit an alignment similar to WMAP. (a): intrinsic (unmodulated) sky from a Gaussian random isotropic realization. (b) (single column): the quadrupolar modulation with $f=-1$ and $w_{2}=-7$ (see (21)) in the dipole direction. (c): the modulated sky of the observed CMB. (d): WMAP full-sky quadrupole and octopole. Adopted from [40].

can definitely be excluded to contribute at significant levels, carbonaceous and silicate dust grains might contribute up to a few $\mu \mathrm{K}$ close to the ecliptic plane, for example, due to the trans-Neptunian object belt. Such an extra contribution along the ecliptic could give rise to $\mathrm{CMB}$ structures aligned with the ecliptic, but those would look very different from the observed ones. On top of that, Solar system dust would be a new additive foreground and could not explain the lack of large-angle correlations. Thus it seems unlikely that Solar system dust grains cause the reported largeangle anomalies, nevertheless they are sources of microwave absorption and emission and may become important to precision measurements in the future.

Finally, it has often been suggested to some of us in private communications that the anomalies may not reflect an unknown foreground that has been neglected, but rather the "missubtraction" of a known foreground. However, it has never quite been clear to us how this leads to the 
observed alignments or lack of large-angle correlations, and we are unaware of any literature that realizes this suggestion successfully.

5.3. Data Analysis Explanations. Most of the results discussed so far have been obtained using reconstructed full-sky maps of the WMAP observations [1, 22, 59]. In the presence of the sky cut of even just a few degrees, the errors in the reconstructed anisotropy pattern, and the directions of multipole vectors, are too large to allow drawing quantitative conclusions about the observed alignments [15]. These large errors are expected: while the power in the $\mathrm{CMB}$ (represented, say, by the angular power spectrum $C_{\ell}$ ) can be accurately recovered since there are $2 \ell+1$ modes available for each $\ell$, there are only 2 modes available for each multipole vector; hence the cut-sky reconstruction is noisier. However the cut-sky alignment probabilities, while very uncertain, are consistent with the full-sky values $[14,50]$; more generally, the alignments appear to be rather robust to Galactic cuts and foreground contamination [60].

A different kind of explanation of missing large-scale power, or missing large-angle correlations, has been taken by Efstathiou et al. [35] who argued that maximum likelihood estimators can be applied to the cut-sky maps to reliably and optimally reconstruct the $\mathrm{CMB}$ anisotropy of the whole sky; for a recent work that extends these ideas, see [33]. This approach yields more two-point correlations on large scales $\left(S_{1 / 2} \sim 8000(\mu K)^{4}\right.$, which is $\sim 5 \%$ likely) than the direct cut-sky pixel-based calculation which gives $S_{1 / 2} \sim$ $1000(\mu K)^{4}$ result and is $\sim 0.03 \%$ likely. These authors then argue that the extremely low $S_{1 / 2}$ obtained by the pixel-based approach applied to the cut-sky is essentially a fluke, and the more reliable result comes from their maximum-likelihood reconstruction of the full-sky. It may indeed be true that the pixel-based calculation is a suboptimal estimate of the full-sky $C(\theta)$ for a statistically isotropic cosmology. However, quantities calculated on the cut-sky are clearly insensitive to assumptions about what lies behind the cut. We can only observe reliably the $\sim 75 \%$ of the sky that was not masked, and that is where the large-angle two-point-correlation is near-vanishing. Any attempt to reconstruct the full-sky must make assumptions about the statistical properties of the CMB sky, and would clearly be affected by the coupling of small-scale and large-scale modes-exactly what is necessary to have a sky in which $S_{1 / 2}$ is anomalously low, while the $C_{\ell}$ 's are individually approximately consistent with the standard cosmology.

5.4. Instrumental Explanations. Are instrumental artifacts the cause of the observed alignments (and/or the low largescale power)? One possible scenario would go as follows. WMAP avoids making observations near the Sun, therefore covering regions away from the ecliptic more than those near the ecliptic. While the corresponding variations in the noise per pixel are well known (e.g., as the number of observations per pixel, $N_{\text {obs }}$; see [1, Figure 3]), and its effects on the largescale anomalies are ostensibly small, they could, in principle, be amplified and create the observed ecliptic anomalies.
However a successful proposal for such an amplification has not yet been put forward.

Another possibility is that an imperfect instrument couples with dominant signals from the sky to create anomalies. Let us review an example given in [40]: suppose that the instrumental response $T_{\text {instr }}(\hat{e})$ to the true sky signal $T(\hat{e})$ is nonlinear

$$
T_{\text {instr }}(\hat{e})=f \sum_{i} \alpha_{i}\left[\frac{T(\hat{e})}{f}\right]^{i}
$$

Here $f$ is an arbitrary normalization scale for the nonlinearity of the response, and $\alpha_{i}$ are arbitrary coefficients with $\alpha_{1}=$ 1. If $\alpha_{i>1} \neq 0$ then $T_{\text {instr }} \neq T$ and the observed temperature is a nonlinear modulation of the true temperature. The dominant temperature signal for a differencing experiment such as WMAP is the dipole arising from our peculiar motion, $T(\hat{e})=T_{\text {dip }} \cos \theta$, with $T_{\text {dip }}=3.35 \mathrm{mK}$ and $\theta$ the polar angle in the dipole frame. Taking $f=T_{\text {dip }}$,

$$
\begin{aligned}
\frac{T_{\text {instr }}(\hat{e})}{T_{\text {dip }}}= & \alpha_{1} P_{1}(\cos \theta)+\alpha_{2}\left[\frac{2}{3 P_{2}(\cos \theta)}+1\right] \\
& +\alpha_{3}\left[\frac{2}{5 P_{3}(\cos \theta)}+\frac{3}{5 P_{1}(\cos \theta)}\right]+\cdots,
\end{aligned}
$$

where $P_{\ell}$ are the Legendre polynomials. Note that with $\alpha_{2} \sim$ $\alpha_{3} \sim O\left(10^{-2}\right)$, the $10^{-3}$ dipole anisotropy is modulated into a $10^{-5}$ quadrupole and octopole anisotropy which are aligned in the dipole frame with the $m=0$ multipole structure. Unfortunately (or fortunately!), WMAP detectors are known to be linear to much better than $1 \%$, so this particular realization of the instrumental explanation does not work. As an aside, note that this type of explanation needs to assume that the higher multipoles are not aligned with the dipole/ecliptic and, moreover, requires essentially no intrinsic power at large scales (that is, even less than what is observed).

To summarize, even though the ecliptic alignments (and the north-south power asymmetry) hint at a systematic effect due to some kind of coupling of an observational strategy and the instrument, to date no plausible proposal of this sort has been put forth.

5.5. Cosmological Explanations. The most exciting possibility is that the observed anomalies have primordial origin, and potentially inform us about the conditions in the early universe. One expects that in this case the alignments with the dipole, or with the solar system, would be statistical flukes.

The breaking of statistical isotropy implies that the usual relation $\left\langle a_{\ell m}^{*} a_{\ell m}\right\rangle=C_{\ell} \delta_{\ell \ell^{\prime}} \delta_{m m^{\prime}}$ does not hold any more; instead

$$
\left\langle a_{\ell m}^{*} a_{\ell m}\right\rangle=C_{\ell \ell^{\prime} m m^{\prime}}
$$

where the detailed form of the quantity on the right-hand side is model dependent (see the more detailed discussions in [61-63]). 
There are many possibilities for how the absence of statistical isotropy might arise; for example, in a nontrivial spatial topology, the fundamental domain would not be rotationally invariant, and so the spherical harmonics (times an appropriate radial function) would not be a basis of independent eigenmodes of the fluctuations. This would certainly lead to a correlation among $a_{\ell m}$ of different $m$ and also $\ell$, although not necessarily to aligned multipoles. The hope would be that the shape of the fundamental domain would lead to these alignments, while a lower density of states at long wavelength (compared to the covering space) would lead to the absence of large-angle correlations. No specific model has been suggested to accomplish all of those, and the matter is complicated by known bounds on cosmic topology [64-68] which force the fundamental domain to be relatively large.

Alternately, in the early universe, asymmetry in the stress-energy tensor of dark energy [69] or a long-wavelength dark energy density mode with a gradient in the desired direction [40], could both imprint the observed alignments via the integrated Sachs-Wolfe mechanism; but it is hard to see how they would explain the lack of large-angle correlations. The authors of [70] have put forward a model where the Sachs-Wolfe contribution to low- $\ell$ multipoles is partly cancelled by the Integrated Sachs-Wolfe contribution, but which still fails to explain the lowness of $S_{1 / 2}$ or the alignments of low- $\ell$ multipoles. Models where the anomalies are caused by breaking of the statistical isotropy $[71,72]$ are especially well studied; see [73] for the equally interesting possibility that the translational invariance is broken.

A commonly used mechanism to explain such anomalies is inflationary models that contain implicit breaking of isotropy [40, 74-80]. Pontzen [81] helpfully shows temperature and polarization patterns caused by various classes of Bianchi models that explicitly break the statistical isotropy. However, outside of explaining the anomalies, the motivation for these anisotropic models is not compelling and they seem somewhat contrived. Moreover, the authors have not investigated whether the low $S_{1 / 2}$ is also observed. Nevertheless, given the large-scale CMB observations, as well as the lack of fundamental theory that would explain inflation, investigating such models is well worthwhile.

A very reasonable approach is to describe breaking of the isotropy with a phenomenological model, measure the parameters of the model, and then try to draw inferences about the underlying physical mechanism. For example, a convenient approach is to describe the breaking of isotropy via the direction-dependent power spectrum of dark matter perturbations [82]

$$
P(\vec{k})=A(k)\left[1+\sum_{\ell m} g_{\ell m}(k) Y_{\ell m}(\hat{k})\right],
$$

where $k=|\vec{k}|, g_{\text {em }}(k)$ quantifies the departure from statistical isotropy as a function of wavenumber $k$, and $A(k)$ refers to the statistically isotropic part of the power spectrum. In this model, the power spectrum, normally considered to depend only on scale $k$, now depends on direction in a parametric way. Statistically significant finding that $g_{\ell m} \neq 0$ for any $(\ell, m)$ would signal a violation of statistical isotropy.

As with the other attempts to explain the anomalies, we conclude that, while there have been some interesting and even promising suggestions, no cosmological explanation to date has been compelling.

5.6. Alignment Explanations: What Next. While future WMAP data is not expected to change any of the observed results, our understanding and analysis techniques are likely to improve. The most interesting test will come from the Planck satellite, whose temperature maps, obtained with a completely different instrument and observational technique than WMAP, could shed significant new light on the alignments. Moreover, polarization information could be extremely useful in distinguishing between different models and classes of explanations in general; for example, Dvorkin et al. [83] explicitly show how polarization information expected from Planck can help identify the cause of the alignments. Finally, one could use the large-scale structure (i.e., galaxy distribution) data on the largest observable scales from surveys such as Dark Energy Survey (DES) and Large Synoptic Survey Telescope (LSST) to test cosmological explanations (see, e.g, $[84,85]$ ).

\section{Explanations from the WMAP Team}

In their seven year data release the WMAP team explicitly discusses several CMB anomalies [46] including the two main ones described in this paper. For the first major issue - the alignment of low multipoles with each otherthe WMAP team agrees that the alignment is observed and arguse, based on work by Francis and Peacock [86], that the integrated Sachs-Wolfe (ISW) contribution of structures at small redshifts $(z \ll 1)$ could be held responsible. There are serious problems with this argument. Firstly, the ordinary Sachs-Wolfe (SW) effect typically dominates at these $\ell$ over the ISW. Thus, only if the ordinary SW effect on the last scattering surface is anomalously low will the ISW contribution dominate. Secondly, though the ISW may lead to alignment of the quadrupole and octopole it is not an explanation for the observed Solar system alignments. This alignment would need to be an additional statistical fluke. Finally, this explanation does nothing whatsoever to mitigate the lack of large scale angular correlation because the ISW effect acts as an additive component and should be statistically uncorrelated from the primordial CMB. Therefore, even if the ISW reconstruction is taken as reliable, this argument would imply

(1) an accidental downward fluctuation of the SW sufficient for the ISW of local structure to dominate and cause an alignment,

(2) an accidental cancellation in angular correlation between the SW and ISW temperature patterns.

Neither the WMAP team nor Francis and Peacock estimate the likelihood of these two newly created puzzles. 
Regarding the second major issue-the lack of angular correlation-the WMAP team refers to a recent work by Efstathiou et al. [35] who argue that quadratic estimators are better estimates of the full-sky from cut-sky data and are in better agreement with the concordance model. While these estimators have been shown to be optimal under the assumption of statistical isotropy, it is unclear why they should be employed when this assumption is to be tested. (For a contrary view, see [33].) The pixel-based estimator applied to the cut-sky in our analysis does not rely on statistical isotropy and it is more conservative as it does not try to reconstruct temperature anisotropies inside the cut. Finally, our claims that the pixel-based cut-sky two-point correlation function is highly anomalous rely on comparisons to the identical correlation function calculated on simulated cut skies. Whether or not it is a good estimate of the fullsky correlation function is answering a different question, (see Section 5.2). Conversely, the estimator suggested in [35] assumes that whatever is within the cut can be reconstructed reliably by truncating the number of multipole moments considered. The latter logic is equivalent to the assumption of the statistical independence of low and high multipoles, which is exactly a consequence of statistical isotropy.

These arguments from the WMAP team offer neither new nor convincing explanations of the observed anomalies discussed in this paper. At best they replace one set of anomalies by another.

\section{Conclusions}

The $\mathrm{CMB}$ is widely regarded as offering strong substantiating evidence for the concordance model of cosmology. Indeed the agreement between theory and data is remarkable-the patterns in the two-point correlation functions (TT, TE, and EE) of Doppler peaks and troughs are reproduced in detail by fitting with only six (or so) cosmological parameters. This agreement should not be taken lightly; it shows our precise understanding of the causal physics on the last scattering surface. Even so, the cosmological model we arrive at is baroque, requiring the introduction at different scales and epochs of three sources of energy density that are only detected gravitationally-dark matter, dark energy and the inflaton. This alone should encourage us to continuously challenge the model and probe the observations particularly on scales larger than the horizon at the time of last scattering.

At the very least, probes of the large-angle $($ low- $\ell$ ) properties of the $\mathrm{CMB}$ reveal that we do not live in a typical realization of the concordance model of inflationary $\Lambda \mathrm{CDM}$. We have reviewed a number of the ways in which that is true: the peculiar geometry of the $\ell=2$ and 3 multipoles-their planarity, their mutual alignment, their alignment perpendicular to the ecliptic and to the dipole; the north-south asymmetry; and the near absence of two-point correlations for points separated by more than $60^{\circ}$.

If indeed the observed $\ell=2$ and $3 \mathrm{CMB}$ fluctuations are not cosmological, one must reconsider all $\mathrm{CMB}$ results that rely on the low $\ell$, for example, the measurement of the optical depth from CMB polarization at low $\ell$ or the spectral index of scalar perturbations and its running. Moreover, the CMB galaxy cross-correlation, which has been used to provide evidence for the Integrated Sachs-Wolfe effect and hence the existence of dark energy, also gets contributions from the lowest multipoles (though the main contribution comes from slightly smaller scales, $\ell \sim 10$ ). Indeed, it is quite possible that the underlying physical mechanism does not cut off abruptly at the octopole, but rather affects the higher multipoles. Indeed, several pieces of evidence have been presented for anomalies at $l>3$ (e.g., $[87,88]$ ). One of these is the parity of the microwave sky. While the observational fact that the octopole is larger than the quadrupole $\left(C_{3}>C_{2}\right)$ is not remarkable on its own, including higher multipoles (up to $\ell \sim 20$ ) the microwave sky appears to be parity odd at a statistically significant level (since WMAP 5yr) [89-91]. It is hard to imagine a cosmological explanation for a parity odd universe, but the same holds true for unidentified systematics or unaccounted astrophysical foregrounds, especially as this recently noticed puzzle shows up in the very well studied angular power spectrum.

While the further WMAP data is not expected to change any of the observed results, our understanding and analysis techniques are likely to improve. Much work remains to study the large-scale correlations using improved foreground treatment, accounting even for the subtle systematics and in particular studying the time-ordered data from the spacecraft. The Planck experiment will be of great importance, as it will provide maps of the largest scales obtained using a very different experimental approach than WMAP-measuring the absolute temperature rather than temperature differences. Polarization maps, when available at high enough signal-to-noise at large scales (which may not be soon), will be a fantastic independent test of the alignments, as each explanation for the alignments, in principle, also predicts the statistics of the polarization pattern on the sky.

\section{Acknowledgment}

D. Huterer is supported by DOE OJI grant under Contract DE-FG02-95ER40899 and NSF under contract AST0807564. D. Huterer and C. J. Copi are supported by NASA under Contract NNX09AC89G; D. J. Schwarz is supported by Deutsche Forschungsgemeinschaft (DFG); G. D. Starkman is supported by a grant from the US Department of Energy; both G. D. Starkman and C. J. Copi are supported by NASA under Cooperative Agreement NNX07AG89G.

\section{References}

[1] C. L. Bennett et al., "First-year Wilkinson Microwave Anisotropy Probe (WMAP) observations: preliminary maps and basic results," The Astrophysical Journal Supplement, vol. 148, no. 1, 2003.

[2] D. N. Spergel, L. Verde, H. V. Peiris et al., "First-year Wilkinson Microwave Anisotropy Probe (WMAP) observations: determination of cosmological parameters," Astrophysical Journal, Supplement Series, vol. 148, no. 1, pp. 175-194, 2003. 
[3] G. Hinshaw, D. N. Spergel, L. Verde et al., "First-year Wilkinson Microwave Anisotropy Probe (WMAP) observations: the angular power spectrum," Astrophysical Journal, Supplement Series, vol. 148, no. 1, pp. 135-159, 2003.

[4] D. N. Spergel, R. Bean, O. Doré et al., "Three-year Wilkinson Microwave Anisotropy Probe (WMAP) observations: implications for cosmology," Astrophysical Journal, Supplement Series, vol. 170, no. 2, pp. 377-408, 2007.

[5] E. Komatsu, J. Dunkley, M. R. Nolta et al., "Five-year wilkinson microwave anisotropy probe observations: cosmological interpretation," Astrophysical Journal, Supplement Series, vol. 180, no. 2, pp. 330-376, 2009.

[6] E. Komatsu et al., "Seven-Year Wilkinson Microwave Anisotropy Probe (WMAP) observations: cosmological interpretation," http://arxiv.org/abs/1001.4538.

[7] D. Huterer, "Mysteries on universe's largest observable scales," New Astronomy Reviews, vol. 50, no. 11-12, pp. 868-874, 2006.

[8] L. R. Abramo and T. S. Pereira, "Testing gaussianity, homogeneity and isotropy with the cosmic microwave background," http://arxiv.org/abs/1002.3173.

[9] C. T. Byrnes and K.-Y. Choi, " Review of local non-Gaussianity from multi-field inflation,” http://arxiv.org/abs/1002.3110.

[10] X. Chen, "Primordial non-Gaussianities from inflation models," http://arxiv.org/abs/1002.1416.

[11] R. K. Sachs and A. M. Wolfe, Astrophysical Journal, vol. 147, p. 73, 1967.

[12] P. J. E. Peebles and D. T. Wilkinson, "Comment on the anisotropy of the primeval fireball," Physical Review, vol. 174, no. 5, p. 2168, 1968.

[13] M. Kamionkowski and L. Knox, "Aspects of the cosmic microwave background dipole," Physical Review D, vol. 67, no. 6, Article ID 063001, 2003.

[14] C. J. Copi, D. Huterer, D. J. Schwarz, and G. D. Starkman, "On the large-angle anomalies of the microwave sky," Monthly Notices of the Royal Astronomical Society, vol. 367, no. 1, pp. 79-102, 2006.

[15] C. J. Copi, D. Huterer, and G. D. Starkman, "Multipole vectors: a new representation of the CMB sky and evidence for statistical anisotropy or non-Gaussianity at $2 \leq \ell \leq 8$," Physical Review D, vol. 70, no. 4, Article ID 043515, 13 pages, 2004.

[16] C. J. Copi, "Freely downloadable multipole vector code," http://www.phys.cwru.edu/projects/mpvectors/\#code.

[17] G. Katz and J. Weeks, "Polynomial interpretation of multipole vectors,” Physical Review D, vol. 70, no. 6, Article ID 063527, 2004.

[18] J. R. Weeks, "Maxwell's multipole vectors and the CMB," http://arxiv.org/abs/astro-ph/0412231.

[19] R. C. Helling, P. Schupp, and T. Tesileanu, "CMB statistical anisotropy, multipole vectors, and the influence of the dipole," Physical Review D, vol. 74, no. 6, Article ID 063004, 2006.

[20] J. C. Maxwell, A Treatise on Electricity and Magnetism, Vol. I, Clarendon Press, London, UK, 3rd edition, 1891.

[21] M. R. Dennis, "Correlations between Maxwell's multipoles for Gaussian random functions on the sphere," Journal of Physics A, vol. 38, no. 8, pp. 1653-1658, 2005.

[22] M. Tegmark, A. de Oliveira-Costa, and A. J. S. Hamilton, "High resolution foreground cleaned CMB map from WMAP,” Physical Review D, vol. 68, no. 12, Article ID 123523, 2003.

[23] A. de Oliveira-Costa, M. Tegmark, M. Zaldarriaga, and A. Hamilton, "Significance of the largest scale CMB fluctuations in WMAP," Physical Review D, vol. 69, no. 6, Article ID 063516, 2004.
[24] D. J. Schwarz, G. D. Starkman, D. Huterer, and C. J. Copi, "Is the low- $\ell$ microwave background cosmic?" Physical Review Letters, vol. 93, no. 22, Article ID 221301, 2004.

[25] C. J. Copi, D. Huterer, D. J. Schwarz, and G. D. Starkman, "Uncorrelated universe: statistical anisotropy and the vanishing angular correlation function in WMAP years 1-3," Physical Review D, vol. 75, no. 2, Article ID 023507, 2007.

[26] H. K. Eriksen, F. K. Hansen, A. J. Banday, K. M. Górski, and P. B. Lilje, "Asymmetries in the cosmic microwave background anisotropy field," Astrophysical Journal, vol. 605, no. 1, pp. 14$20,2004$.

[27] F. K. Hansen, P. Cabella, D. Marinucci, and N. Vittorio, "Asymmetries in the local curvature of the wilkinson microwave anisotropy probe data," Astrophysical Journal, vol. 607, no. 2, pp. L67-L70, 2004.

[28] A. Bernui, T. Villela, C. A. Wuensche, R. Leonardi, and I. Ferreira, "On the cosmic microwave background large-scale angular correlations," Astronomy and Astrophysics, vol. 454, no. 2, pp. 409-414, 2006.

[29] A. Bernui, B. Mota, M. J. Rebouças, and R. Tavakol, "Mapping the large-scale anisotropy in the WMAP data," Astronomy and Astrophysics, vol. 464, no. 2, pp. 479-485, 2007.

[30] M. R. Nolta, J. Dunkley, R. S. Hill et al., "Five-year wilkinson microwave anisotropy probe observations: angular power spectra," Astrophysical Journal, Supplement Series, vol. 180, no. 2, pp. 296-305, 2009.

[31] C. J. Copi, D. Huterer, D. J. Schwarz, and G. D. Starkman, "No large-angle correlations on the non-Galactic microwave sky," Monthly Notices of the Royal Astronomical Society, vol. 399, no. 1, pp. 295-303, 2009.

[32] G. Hinshaw, A. J. Banday, C. L. Bennett et al., "Two-point correlations in the COBE 1 DMR four-year anisotropy maps," Astrophysical Journal, vol. 464, no. 1, pp. L25-L28, 1996.

[33] A. Pontzen and H. V. Peiris, "The cut-sky cosmic microwave background is not anomalous," Physical Review D, vol. 81, no. 10, Article ID 103008, 2010.

[34] A. Hajian, "Analysis of the apparent lack of power in the cosmic microwave background anisotropy at large angular scales," http://arxiv.org/abs/astro-ph/0702723.

[35] G. Efstathiou, Y.-Z. Ma, and D. Hanson, "Large-angle correlations in the cosmic microwave background," Monthly Notices of the Royal Astronomical Society, vol. 407, no. 3, pp. 25302542, 2010.

[36] G. Efstathiou, "A maximum likelihood analysis of the low cosmic microwave background multipoles from the Wilkinson Microwave Anisotropy Probe," Monthly Notices of the Royal Astronomical Society, vol. 348, no. 3, pp. 885-896, 2004.

[37] I. J. O'Dwyer, H. K. Eriksen, B. D. Wandelt et al., "Bayesian power spectrum analysis of the first-year Wilkinson Microwave Anisotropy Probe data," Astrophysical Journal, vol. 617, no. 2, pp. L99-L102, 2004.

[38] A. Rakić and D. J. Schwarz, "Correlating anomalies of the microwave sky," Physical Review D, vol. 75, no. 10, Article ID 103002, 2007.

[39] D. Sarkar, D. Huterer, C. J. Copi, G. D. Starkman, and D. J. Schwarz, "Missing power vs low-l alignments in the cosmic microwave background: no correlation in the standard cosmological model," http://arxiv.org/abs/1004.3784.

[40] C. Gordon, W. Hu, D. Huterer, and T. Crawford, "Spontaneous isotropy breaking: a mechanism for CMB multipole alignments," Physical Review D, vol. 72, no. 10, Article ID 103002, 13 pages, 2005.

[41] E. F. Bunn and A. Bourdon, "Contamination cannot explain the lack of large-scale power in the cosmic microwave 
background radiation," Physical Review D, vol. 78, no. 12, Article ID 123509, 2008.

[42] N. E. Groeneboom, H. K. Eriksen, K. Gorski, G. Huey, J. Jewell, and B. Wandelt, "Bayesian analysis of white noise levels in the five-year WMAP data," Astrophysical Journal, vol. 702, no. 1, pp. L87-L90, 2009.

[43] N. E. Groeneboom and H. K. Eriksen, "Bayesian analysis of sparse anisotropic universe models and application to the fiveyear WMAP data," Astrophysical Journal, vol. 690, no. 2, pp. 1807-1819, 2009.

[44] D. Hanson and A. Lewis, "Estimators for CMB statistical anisotropy," Physical Review D, vol. 80, no. 6, Article ID 063004, 2009.

[45] L. Ackerman, S. M. Carroll, and M. B. Wise, "Imprints of a primordial preferred direction on the microwave background," Physical Review D, vol. 75, no. 8, Article ID 083502, 2007.

[46] C. L. Bennett et al., " Seven-year Wilkinson Microwave Anisotropy Probe (WMAP) observations: are there cosmic microwave background anomalies?" http://arxiv.org/abs/1001.4758.

[47] D. Hanson, A. Lewis, and A. Challinor, "Asymmetric beams and CMB statistical anisotropy," Physical Review D, vol. 81, no. 10, Article ID 103003, 2010.

[48] J. Hoftuft, H. K. Eriksen, A. J. Banday, K. M. Górski, F. K. Hansen, and P. B. Lilje, "Increasing evidence for hemispherical power asymmetry in the five-year wmap data," Astrophysical Journal, vol. 699, no. 2, pp. 985-989, 2009.

[49] S. Bashinsky and U. Seljak, "Signatures of relativistic neutrinos in CMB anisotropy and matter clustering," Physical Review D, vol. 69, no. 8, Article ID 083002, 35 pages, 2004.

[50] P. Bielewicz, H. K. Eriksen, A. J. Banday, K. M. Górski, and P. B. Lilje, "Multipole vector anomalies in the first-year WMAP data: a cut-sky analysis," Astrophysical Journal, vol. 635, no. 2, pp. 750-760, 2005.

[51] A. Rakić, S. Räsänen, and D. J. Schwarz, Monthly Notices of the Royal Astronomical Society, vol. 369, pp. L27-L31, 2006.

[52] P. C. Frisch, "Tentative identification of interstellar dust in the magnetic wall of the heliosphere," Astrophysical Journal, vol. 632, no. 2, pp. L143-L146, 2005.

[53] K. T. Indue and J. Silk, "Local voids as the origin of large-angle cosmic microwave background anomalies. I," Astrophysical Journal, vol. 648, no. 1, pp. 23-30, 2006.

[54] H. V. Peiris and T. L. Smith, "CMB isotropy anomalies and the local kinetic Sunyaev-Zel'dovich effect," Physical Review D, vol. 81, no. 12, Article ID 123517, 2010.

[55] C. Vale, "Local pancake defeats axis of evil," http://arxiv.org/abs/astro-ph/0509039.

[56] A. Cooray and N. Seto, "Did the Wilkinson Microwave Anisotropy Probe see moving local structures?" Journal of Cosmology and Astroparticle Physics, vol. 2005, no. 12, article 4, pp. 41-49, 2005.

[57] V. Dikarev, O. Preuß, S. Solanki, H. Krüger, and A. Krivov, "Understanding the WMAP results: low-order multipoles and dust in the vicinity of the solar system," Earth, Moon and Planets, vol. 102, no. 1-4, pp. 555-561, 2008.

[58] V. Dikarev, O. Preuß, S. Solanki, H. Krüger, and A. Krivov, "The local dust foregrounds in the microwave sky. I. thermal emission spectra," Astrophysical Journal, vol. 705, no. 1, pp. 670-682, 2009.

[59] H. K. Eriksen, A. J. Banday, K. M. Górski, and P. B. Lilje, "On foreground removal from the wilkinson microwave anisotropy probe data by an internal linear combination method: limitations and implications," Astrophysical Journal, vol. 612, no. 2, pp. 633-646, 2004.
[60] A. de Oliveira-Costa and M. Tegmark, "CMB multipole measurements in the presence of foregrounds," Physical Review D, vol. 74, no. 2, Article ID 023005, 2006.

[61] A. Hajian and T. Souradeep, "Measuring the statistical isotropy of the cosmic microwave background anisotropy," Astrophysical Journal, vol. 597, no. 1, pp. L5-L8, 2003.

[62] A. Hajian, T. Souradeep, and N. Cornish, "Statistical isotropy of the wilkinson microwave anisotropy probe data: a bipolar power spectrum analysis," Astrophysical Journal, vol. 618, no. 2, pp. L63-L66, 2005.

[63] T. S. Pereira and L. R. Abramo, "Angular-planar CMB power spectrum," Physical Review D, vol. 80, no. 6, Article ID 063525 , 2009.

[64] N. J. Cornish, D. N. Spergel, and G. D. Starkman, "Measuring the topology of the universe," Proceedings of the National Academy of Sciences of the United States of America, vol. 95, no. 1, pp. 82-84, 1998.

[65] N. J. Cornish, D. Spergel, and G. Starkman, "Can COBE see the shape of the universe?" Physical Review D, vol. 57, no. 10, pp. 5982-5996, 1998.

[66] N. J. Cornish, D. N. Spergel, and G. D. Starkman, "Circles in the sky: finding topology with the microwave background radiation," Classical and Quantum Gravity, vol. 15, no. 9, pp. 2657-2670, 1998.

[67] N. J. Cornish, D. N. Spergel, G. D. Starkman, and E. Komatsu, "Constraining the topology of the Universe," Physical Review Letters, vol. 92, no. 20, Article ID 201302, 2004.

[68] J. S. Key, N. J. Cornish, D. N. Spergel, and G. D. Starkman, "Extending the WMAP bound on the size of the Universe," Physical Review D, vol. 75, no. 8, Article ID 084034, 2007.

[69] R. Battye and A. Moss, "Anisotropic dark energy and CMB anomalies," Physical Review D, vol. 80, no. 2, Article ID 023531, 2009.

[70] N. Afshordi, G. Geshnizjani, and J. Khoury, "Do observations offer evidence for cosmological-scale extra dimensions?" Journal of Cosmology and Astroparticle Physics, vol. 2009, no. 8, article 30, 2009.

[71] T. R. Jaffe, A. J. Banday, H. K. Eriksen, K. M. Górski, and F. K. Hansen, "Evidence of vorticity and shear at large angular scales in the WMAP data: a violation of cosmological isotropy?" Astrophysical Journal, vol. 629, no. 1, pp. L1-L4, 2005.

[72] T. Ghosh, A. Hajian, and T. Souradeep, "Unveiling hidden patterns in CMB anisotropy maps," Physical Review D, vol. 75, no. 8, Article ID 083007, 2007.

[73] S. M. Carroll, C.-Y. Tseng, and M. B. Wise, "Translational invariance and the anisotropy of the cosmic microwave background," Physical Review D, vol. 81, no. 8, Article ID 083501, 2010.

[74] L. Ackerman, S. M. Carroll, and M. B. Wise, "Imprints of a primordial preferred direction on the microwave background," Physical Review D, vol. 75, no. 8, Article ID 083502, 2007.

[75] C. Armendariz-Picon, "Creating statistically anisotropic and inhomogeneous perturbations," Journal of Cosmology and Astroparticle Physics, vol. 2007, no. 9, article 14, 2007.

[76] A. E. Gümrükçüoğlu, C. R. Contaldi, and M. Peloso, "Inflationary perturbations in anisotropic backgrounds and their imprint on the cosmic microwave background," Journal of Cosmology and Astroparticle Physics, vol. 2007, no. 11, article 5, 2007.

[77] D. C. Rodrigues, "Anisotropic cosmological constant and the CMB quadrupole anomaly," Physical Review D, vol. 77, no. 2, Article ID 023534, 2008. 
[78] C. Pitrou, T. S. Pereira, and J.-P. Uzan, "Predictions from an anisotropic inflationary era," Journal of Cosmology and Astroparticle Physics, vol. 2008, no. 4, article 4, 2008.

[79] A. L. Erickcek, M. Kamionkowski, and S. M. Carroll, "A hemispherical power asymmetry from inflation," Physical Review D, vol. 78, no. 12, Article ID 123520, 2008.

[80] A. L. Erickcek, C. M. Hirata, and M. Kamionkowski, "A scale-dependent power asymmetry from isocurvature perturbations," Physical Review D, vol. 80, no. 8, Article ID 083507, 2009.

[81] A. Pontzen, "Rogues' gallery: the full freedom of the Bianchi CMB anomalies," Physical Review D, vol. 79, no. 10, Article ID 103518, 2009.

[82] A. R. Pullen and M. Kamionkowski, "Cosmic microwave background statistics for a direction-dependent primordial power spectrum," Physical Review D, vol. 76, no. 10, Article ID 103529, 2007.

[83] C. Dvorkin, H. V. Peiris, and W. Hu, "Testable polarization predictions for models of CMB isotropy anomalies," Physical Review D, vol. 77, no. 6, Article ID 063008, 2008.

[84] H. Zhan, L. Knox, J. A. Tyson, and V. Margoniner, "Exploring large-scale structure with billions of galaxies," Astrophysical Journal, vol. 640, no. 1, pp. 8-17, 2006.

[85] A. R. Pullen and C. M. Hirata, "Non-detection of a statistically anisotropic power spectrum in large-scale structure," Journal of Cosmology and Astroparticle Physics, vol. 2010, no. 5, article 27, 2010.

[86] C. L. Francis and J. A. Peacock, "An estimate of the local integrated Sachs-Wolfe signal and its impact on cosmic microwave background anomalies," Monthly Notices of the Royal Astronomical Society, vol. 406, no. 1, pp. 14-21, 2010.

[87] K. Land and J. Magueijo, "Examination of evidence for a preferred axis in the cosmic radiation anisotropy," Physical Review Letters, vol. 95, no. 7, Article ID 071301, 4 pages, 2005.

[88] K. Land and J. Magueijo, "The axis of evil revisited," Monthly Notices of the Royal Astronomical Society, vol. 378, no. 1, pp. 153-158, 2007.

[89] K. Land and J. Magueijo, "Is the Universe odd?" Physical Review D, vol. 72, no. 10, Article ID 101302, 5 pages, 2005.

[90] J. Kim and P. Naselsky, "Anomalous parity asymmetry of the Wilkinson microwave anisotropy probe power spectrum data at low multipoles," Astrophysical Journal, vol. 714, no. 2, pp. L265-L267, 2010.

[91] J. Kim and P. Naselsky, "Anomalous parity asymmetry of WMAP power spectrum data at low multpoles: is it cosmological or systematics?" http://arxiv.org/abs/1002.0148. 

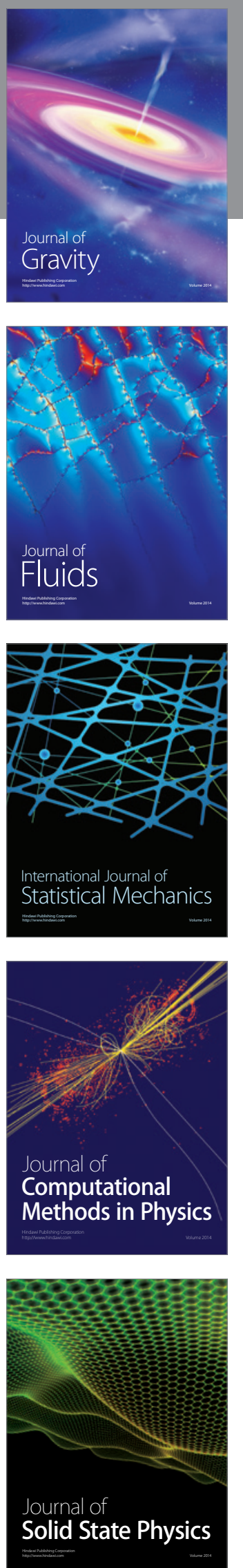

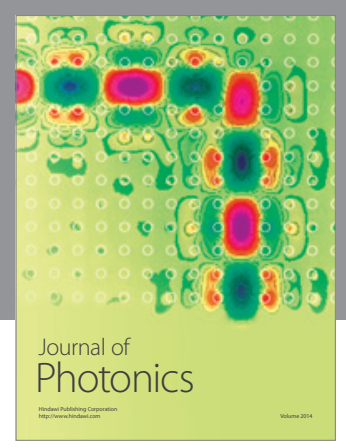

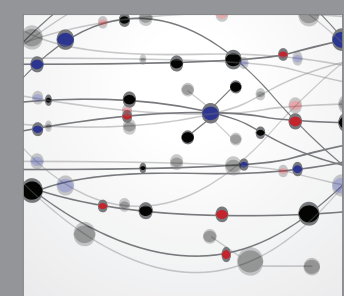

The Scientific World Journal
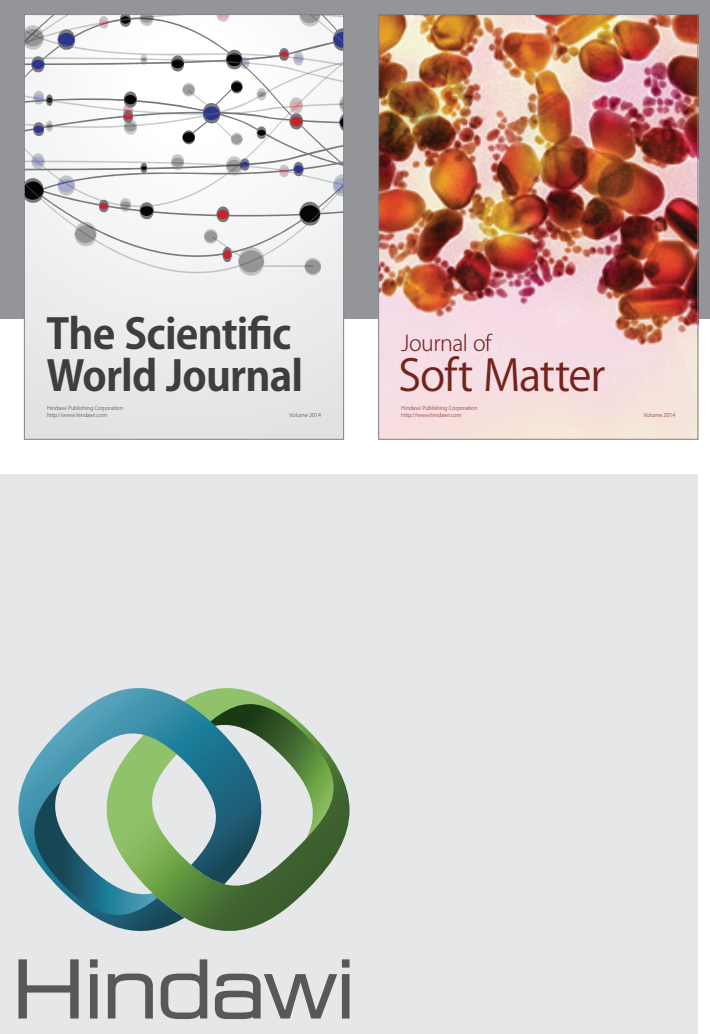

Submit your manuscripts at

http://www.hindawi.com
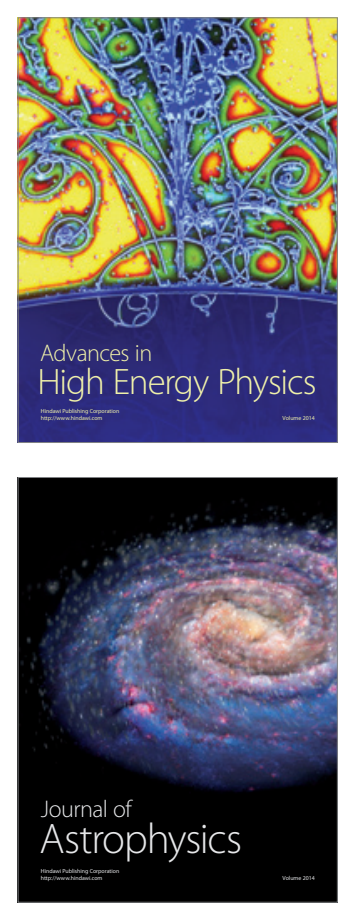
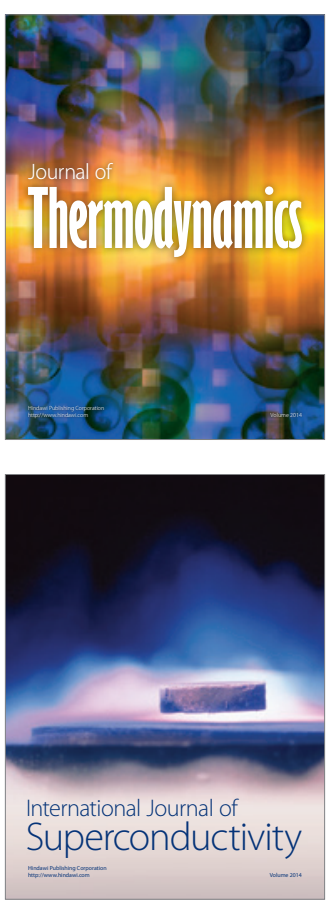
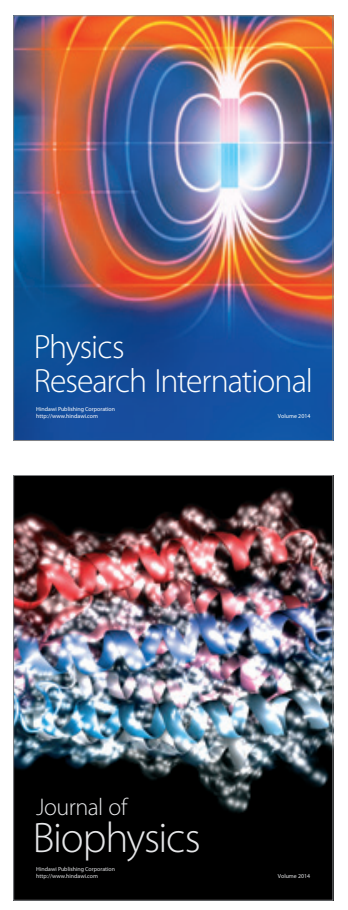
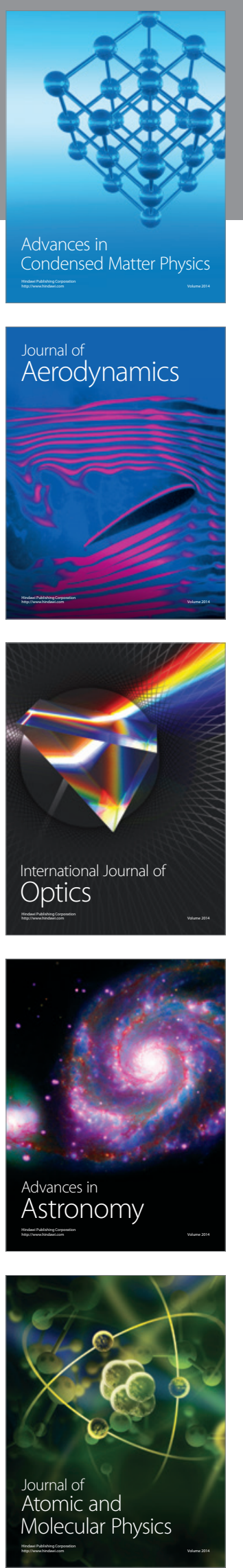